ARTICLE

Received 29 Jun 2015 | Accepted 4 Jan 2016 | Published 3 Feb $2016 \quad$ DOl: 10.1038/ncomms10597 OPEN

\title{
NuSAP governs chromosome oscillation by facilitating the Kid-generated polar ejection force
}

\author{
Chenyu Li ${ }^{1, \dagger}$, Chenyi Xue ${ }^{2}$, Qiaoyun Yang ${ }^{1}$, Boon Chuan Low ${ }^{1,3} \&$ Yih-Cherng Liou ${ }^{1,4}$
}

In vertebrate cells, chromosomes oscillate to align precisely during metaphase. NuSAP, a microtubule-associated protein, plays a critical role in stabilizing spindle microtubules. In this study, we utilize 3D time-lapse live-cell imaging to monitor the role of NuSAP in chromosome oscillation and identify NuSAP as a novel regulator of the chromokinesin, Kid. Depletion of NuSAP significantly suppresses the amplitude and velocity of chromosome oscillation. We analyse the effects of NuSAP and Kid depletion in monopolar and bipolar cells with or without kinetochore microtubule depletion. Twelve postulated conditions are deciphered to reveal the contribution of NuSAP to the polar force generated at kinetochore microtubules and to the regulation of the polar ejection force generated by Kid, thus revealing a pivotal role of NuSAP in chromosome oscillation.

\footnotetext{
${ }^{1}$ Department of Biological Sciences, Faculty of Science, National University of Singapore, 14 Science Drive 4, Singapore 117543, Republic of Singapore. ${ }^{2}$ Cardiovascular Institute, Perelman School of Medicine, University of Pennsylvania, Philadelphia, Pennsylvania 19104, USA. ${ }^{3}$ Mechanobiology Institute, National University of Singapore, Singapore 117411, Republic of Singapore. ${ }^{4}$ Graduate School for Integrative Sciences and Engineering, National University of Singapore, Singapore 117573, Republic of Singapore. † Present address: Harvard Medical School, Center for Life Science 0428, Beth Israel Deaconess Medical Center, Boston, Massachusetts 02215, USA. Correspondence and requests for materials should be addressed to Y.-C.L. (email: dbslyc@nus.edu.sg).
} 
C hromosome oscillation is a common feature of metaphase in most eukaryotic cells ${ }^{1}$. The movement of chromosomes can be polewards or anti-polewards, referring to the direction of movement towards the pole or away from the pole ${ }^{2}$. Poleward motion is produced by the polar force (PF), which is predominantly generated by the depolymerization of kinetochore microtubules $(\mathrm{kMTs})^{3}$, while anti-poleward motion is produced by the polar ejection force (PEF), which is dependent on motor proteins sliding along the chromosome arms at interpolar microtubules (iMTs) ${ }^{4}$. Although a number of studies have focused on two-dimensional kinetochore (KT) behaviour ${ }^{5-8}$ and the biophysical prediction of KT movement ${ }^{9-12}$, the underlying molecular mechanism of KT oscillation is still largely unknown.

Microtubule-associated proteins (MAPs) play vital roles in regulating chromosome oscillation by tightly maintaining both the dynamics of kMTs and the surface properties of iMTs ${ }^{13}$. NuSAP (Nucleolar and Spindle-Associated Protein) ${ }^{14}$, a RanGTP-regulated MAP, bundles microtubules ${ }^{15}$ and links them to chromosomes ${ }^{16}$. In addition, NuSAP regulates spindle assembly, chromosome segregation and cytokinesis ${ }^{14,17}$. The level of NuSAP protein expression is tightly regulated during the cell cycle by anaphase-promoting complex/cyclosome $e^{18,19}$ and is upregulated in several types of cancer ${ }^{20-25}$. However, the function of NuSAP in chromosome oscillation has not yet been elucidated.

Human chromokinesins are plus-end-directed motors contributing to anti-poleward movement ${ }^{26-28}$. These include Kid (kinesin-like DNA-binding protein), which contains an $\mathrm{N}$-terminal microtubule-binding domain and a C-terminal chromosome-interacting domain ${ }^{29}$. Kid functions as a microtubule-based motor, generating the PEF, and regulating the orientation of chromosome arms and KT oscillation ${ }^{7,8,30}$. The RanGTP gradient, which regulates NuSAP localization, promotes the accumulation of Kid on chromosomes ${ }^{31}$. Although a functional relationship has been reported between NuMA and Kid in spindle morphology and chromosome alignment ${ }^{32}$, and the microtubule localization of Kid is known to be mediated by the spindle protein $\mathrm{CHICA}^{33}$, the regulatory mechanism of Kid in chromosome oscillation remains unclear.

In this study, we sought to determine the role of NuSAP in chromosome oscillation. We use three-dimensional (3D) timelapse live-cell imaging to analyse chromosome oscillation in a dynamically heterogeneous population to determine the influence of NuSAP on the Kid-generated PEF. Our results show that NuSAP plays a pivotal role in mediating chromosome oscillation through its regulation on the Kid-generated PEF during metaphase.

\section{Results}

NuSAP regulates chromosome alignment and orientation. To determine the function of NuSAP during metaphase, we first investigated the localization of GFP-NuSAP along the spindle using fluorescent imaging (Fig. 1a). Line graphs showing the intensity of signal across the spindle pole showed that NuSAP predominantly localized at the central spindle microtubules. NuSAP-overexpressing cells were also found to display a large proportion of misaligned chromosomes (Fig. 1b). To quantify the level of misalignment, we applied the index of chromosome alignment $^{7}$, which calculates the ratio of the fluorescence of anticentromere antibody staining in the central spindle compared with the whole spindle (Fig. 1b). The index of chromosome alignment of GFP-NuSAP-overexpressing cells was significantly smaller $(0.65 \pm 0.07, \pm$ s.d. from three independent experiments) than that of the control cells $(0.94 \pm 0.03)$, indicating a severe chromosome misalignment phenotype (Fig. 1c), which suggests that NuSAP may have a role in regulating chromosome alignment.
To determine how NuSAP disturbs chromosome congression, we used $3 \mathrm{D}$ time-lapse live-cell imaging to monitor the movement of chromosomes in synchronized HeLa cells stably expressing mCherry-H2B (Fig. 1d and Supplementary Movie 1). Strikingly, we found that chromosomes in cells overexpressing NuSAP displayed prominent misorientation, with the arms parallel rather than perpendicular to the spindle axis (Fig. 1d, row 1). Selected misaligned chromosomes are presented as a $3 \mathrm{D}$ reconstruction (row 2). The time-lapse projection for the lagging chromosome showed that the arms of the chromosome were rotating and stretching over time (Fig. 1e), suggesting that unbalanced forces on the arms were disrupting the movement of the chromosomes. Thus, upregulated NuSAP protein levels results in an imbalance of forces on the chromosome arms, resulting in chromosome misorientation.

NuSAP interacts with Kid and regulates its localization. The faulty orientation of chromosomes in NuSAP-overexpressing cells suggests unbalanced forces on the chromosome arms. The configuration of the chromosome pushing away from the spindle poles in Drosophila cells was identified as the elevation of $\mathrm{PEF}^{34}$. Previous studies indicate that Kid, a chromokinesin, is essential for generating the PEF at chromosome arms during chromosome oscillation $^{5,8}$. This observation motivated us to investigate the interaction between NuSAP and Kid. Immunoprecipitation experiments indicated that NuSAP interacted with Kid endogenously (Fig. 2a and Supplementary Fig. 1a,b). Using purified recombinant His-tagged NuSAP and glutathione $S$-transferase (GST)-tagged Kid proteins, we confirmed this interaction by demonstrating that NuSAP could be pulled down by Kid in vitro (Fig. 2b). To determine the temporal nature of this interaction during the mitotic phase, cells were first arrested using thymidine/nocodazole treatments and then released into fresh medium (Fig. 2c). Immunoprecipitation at various time points post release indicated that the interaction of NuSAP with Kid occurred specifically at metaphase (Fig. 2c, lane 1).

The PEF generated by Kid during metaphase largely depends on the association of Kid with iMTs ${ }^{29,35}$. As NuSAP primarily localizes to the central spindle microtubules, we questioned whether the interaction between NuSAP and Kid affects the localization of Kid on microtubules. To address this question, we first examined the localization of endogenous Kid and NuSAP on mitotic spindles. Both Kid and NuSAP were observed to concentrate in the middle spindle (Supplementary Fig. 1c). NuSAP overexpression was found to disrupt the distribution of Kid along the spindle, with an increased fluorescent intensity along the microtubules in monopolar and metaphase cells (Fig. 2d). This observation was confirmed using live cells expressing mCherry-Kid (Supplementary Fig. 1d). Overexpression of NuSAP increased the association of Kid with microtubules, while depletion of NuSAP caused Kid to relocalize to chromosomes. To rule out the possibility of a dominantnegative effect, GFP-vector or GFP-NuSAP was expressed in either control short interfering RNA (siRNA)- or NuSAP siRNA-depleted cells (Fig. 2e). The microtubule localization of Kid could be rescued by overexpression of NuSAP (Fig. 2e, row 4). Taken together, our results indicate that NuSAP interacts with Kid at metaphase and enhances the association between Kid and microtubules.

The depletion of NuSAP or Kid attenuates centromere movement. To understand how NuSAP regulates Kid function in chromosome oscillation, 3D time-lapse live-cell imaging was conducted with GFP-CENPA-labelled centromeres in synchronized HeLa cells stably expressing mCherry-H2B. Cells were transfected with 
a
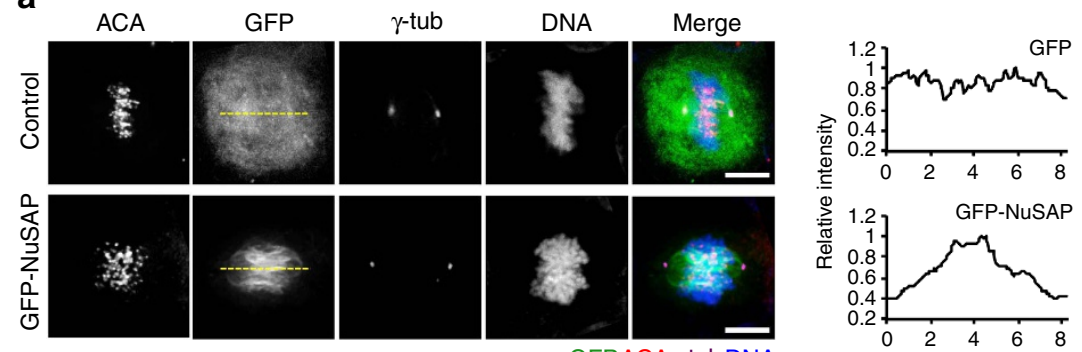

b
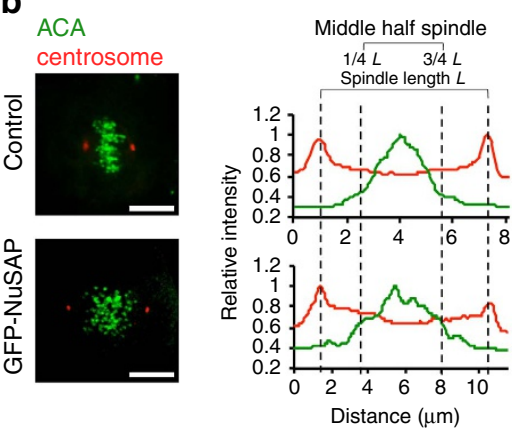

C

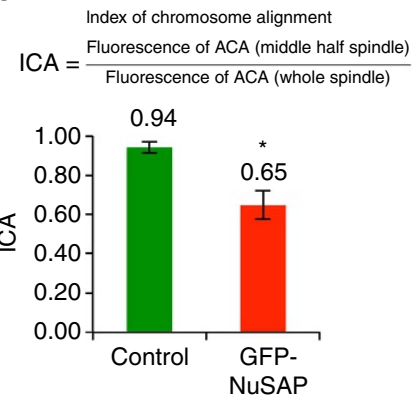

d
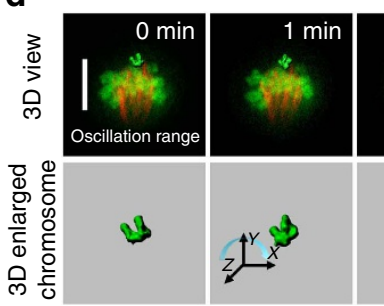

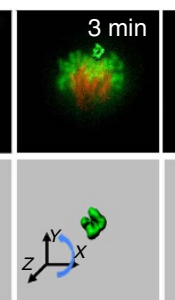

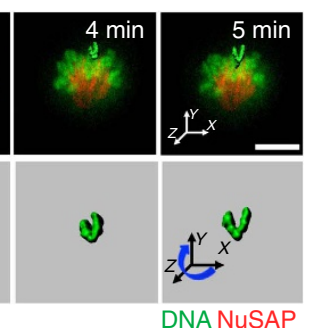

e

Chromosome time project

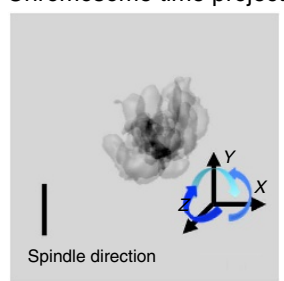

Figure 1 | NuSAP regulates chromosome alignment and orientation during metaphase. (a) Fluorescent images of synchronized metaphase HeLa cells expressing GFP-NuSAP and GFP-vector (control). The line profiles of the GFP channel are presented in the graph on the right. Mitotic spindles were labelled with anti- $\gamma$-tubulin and anti-CREST, and DNA with Hoechst 333342. Scale bar, $5 \mu \mathrm{m}$. (b) Representative images using the ICA method to analyse centromere alignment in HeLa cells expressing GFP-NuSAP and GFP-vector only (control), with data represented as a line graph on the right. The distribution of ACA fluorescence within the spindle region was measured along the spindle axis following staining with anti- $\gamma$-tubulin and anti-Crest. (c) ICAs in HeLa cells expressing GFP-NuSAP and GFP-vector only (control) were analysed as in $\mathbf{b}$. Data were collected from three independent experiments and error bars represent \pm s.d. ${ }^{*} P<0.001$, by $t$-test. (d) 3D time-lapse imaging and 3D single chromosome surface of chromosome oscillation in synchronized metaphase mCherry-H2B HeLa cells stably expressing GFP-NuSAP. Changes in the chromosome orientation are indicated by arrows. Scale bar, $5 \mu \mathrm{m}$. (e) Time project image representing the single chromosome misorientation.

control, NuSAP (Supplementary Fig. 2a-c) or Kid siRNA (Supplementary Fig. 3a) for $48 \mathrm{~h}$ and the metaphase cells were imaged. Representative 3D images using $x z$ and $y z$ projections are shown in Fig. 3a and Supplementary Movie 2. To gain insight into the KT dynamics, the centromere positions were detected and tracked in $3 \mathrm{D}$ over time during oscillation, maximizing the signal-to-noise ratio and minimizing phototoxicity ${ }^{36}$. The tracks were colour-coded over time (Fig. 3b, lane 1). Consistent with previous work ${ }^{7,8}$, the centromeres in Kid-depleted cells exhibited a slower velocity, with most tracks shown in blue and green $\left(0-2 \mu \mathrm{m} \mathrm{min}^{-1}\right)$ and fewer in red $\left(2-3 \mu \mathrm{m} \mathrm{min}^{-1}\right)$, when colourcoded for velocity, compared with the control cells (Fig. 3b, lane 2). The majority of centromere velocities in NuSAP-depleted cells were also in the $0-2 \mu \mathrm{m} \mathrm{min}^{-1}$ range, a similar pattern to that of Kid-depleted cells (Fig. 3b, lane 2), indicating that depletion of either NuSAP or Kid results in a decreased velocity of chromosome oscillation in metaphase cells.

To further investigate the function of NuSAP and Kid in chromosome movement, we characterized the intercentromere distance (ICD) of sister centromeres and the half-period of the 3D time-lapse tracks (Table 1). The ICDs in the NuSAP-depleted $(0.69 \pm 0.09 \mu \mathrm{m})$ and Kid-depleted $(0.71 \pm 0.10 \mu \mathrm{m})$ cells were not significantly changed compared with that of the control cells $(0.75 \pm 0.09 \mu \mathrm{m})$. The average half-periods also displayed similar ranges with $32.50 \pm 3.34 \mathrm{~s}$ for the control siRNA cells, $32.56 \pm 3.55 \mathrm{~s}$ for the NuSAP-depleted cells and $31.73 \pm 4.14 \mathrm{~s}$ for the Kid-depleted cells (Table 1), suggesting that the stiffness between the sister centromeres was not greatly affected by the depletion of NuSAP or Kid. To investigate the effects of NuSAP and Kid depletion on the kinetic features of the oscillatory activity, we set the projected $3 \mathrm{D}$ displacement of the centre in a sister centromere pair as the centre point tracks over a time course to show the oscillation of a particular chromosome $e^{11,36}$ As shown in Fig. $3 c$, and consistent with previous reports ${ }^{7,8,32}$, the oscillation amplitude of the centre in Kid-depleted cells was dramatically decreased $(0.79 \pm 0.05 \mu \mathrm{m})$ compared with the control cells $(1.12 \pm 0.08 \mu \mathrm{m})$. Similarly, the oscillation amplitude in NuSAP-depleted cells was reduced to $0.78 \pm 0.11 \mu \mathrm{m}$ (Fig. $3 \mathrm{c}$ ). 
a

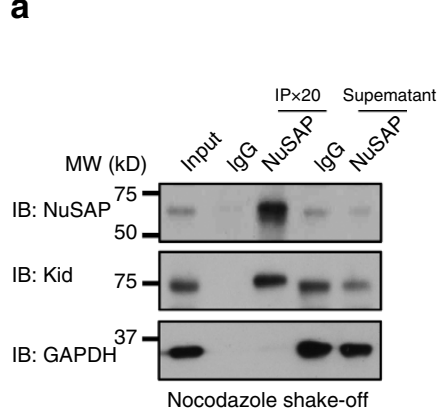

b

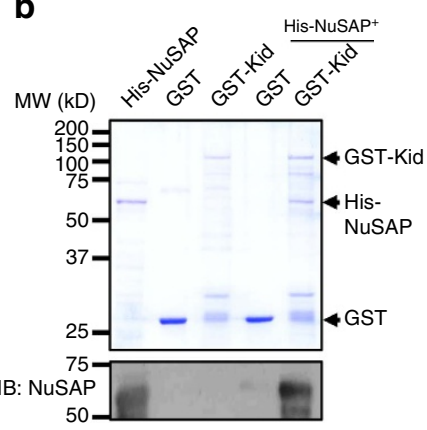

C

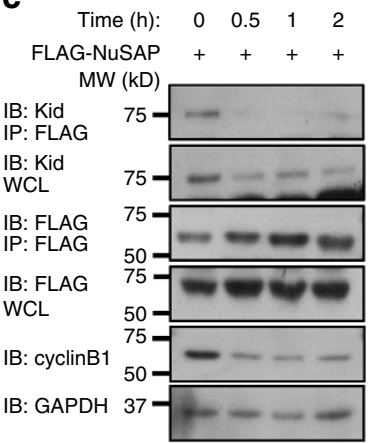

d

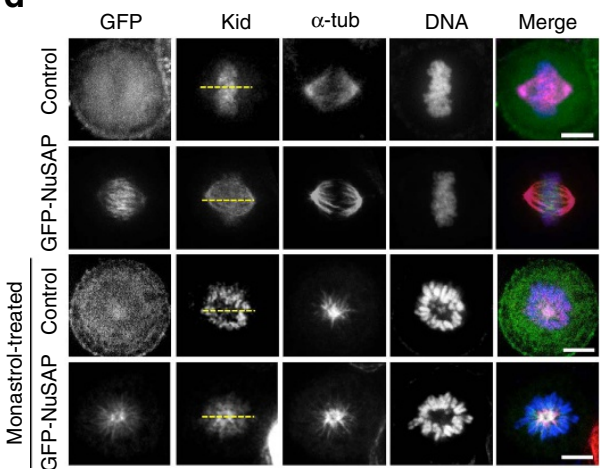

Kid GFP $\alpha$-tub DNA

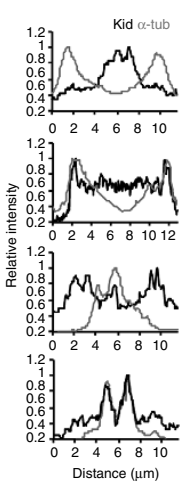

e
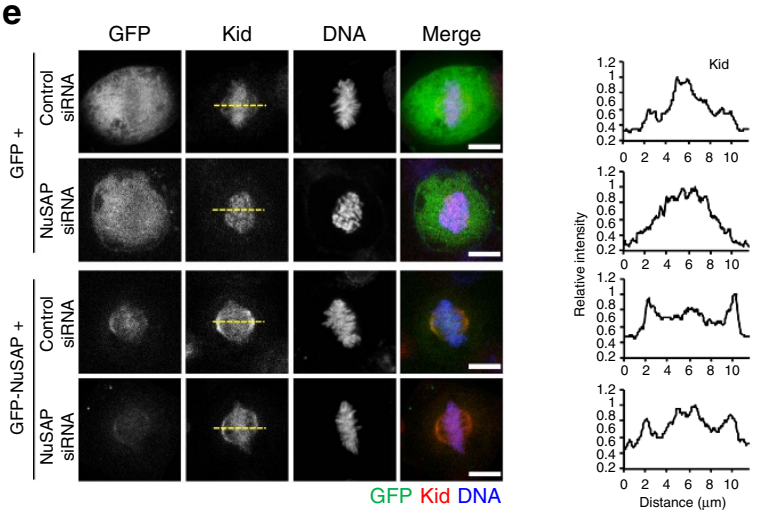

Figure 2 | NuSAP binds Kid and regulates its localization at microtubules. (a) NuSAP binds Kid endogenously. Synchronized HeLa cell lysates were incubated with $2 \mu \mathrm{g}$ rabbit IgG or NuSAP antibody bound to protein A-Sepharose beads. The immunoprecipitated proteins and supernatants were blotted with anti-Kid, anti-NuSAP and anti-GAPDH antibodies. (b) NuSAP binds Kid in vitro. Purified His-NuSAP recombinant protein was incubated with either GST or GST-Kid recombinant protein. GST was pulled down and NuSAP was blotted with an anti-NuSAP antibody. (c) Immunoprecipitation of NuSAP with Kid was conducted with whole-cell lysates of HeLa cells transfected with FLAG-NuSAP after release from thymidine/nocodazole arrest into fresh medium at the indicated time points. The immunoprecipitated protein and the whole-cell lysates were blotted with anti-Kid, anti-FLAG, anti-cyclin B1 and anti-GAPDH antibodies. (d) Kid localization at the kinetochore region in bipolar metaphase HeLa cells and monastrol-treated monopolar cells expressing GFP-NuSAP and the GFP-vector (control). Cells were stained with anti- $\alpha$-tubulin and Hoechst 33342. The line profile of Kid localization (black) according to $\alpha$-tubulin (grey) is represented in the right-hand graph. Scale bar, $5 \mu \mathrm{m}$. (e) Kid localization in bipolar metaphase HeLa cells transfected with control siRNA or NuSAP siRNA together with GFP-vector or GFP-NuSAP. The line profile of Kid localization (black) is represented in the right-hand graph.

Scale bar, $5 \mu \mathrm{m}$.

In addition, the distribution of the amplitude in the NuSAP or Kid-depleted cells showed an increase in the lower range $(0-1 \mu \mathrm{m})$ and a decrease in the larger range $(1-2 \mu \mathrm{m})$ compared with the control cells (Fig. 3d). As the amplitude of the oscillation is directly correlated with the $\mathrm{PEF}^{37}$, the significant decrease in the amplitude in the NuSAP- and Kid-depleted cells suggests a general reduction of the PEF. To analyse the dynamics of the chromosome during oscillation further, the velocity of the projected centre point in control, NuSAP- or Kid-depleted cells was also calculated. As shown in Fig. 3e, the depletion of either
NuSAP $\left(1.39 \pm 0.03 \mu \mathrm{m} \mathrm{min}^{-1}\right)$ or Kid $\left(1.54 \pm 0.06 \mu \mathrm{m} \mathrm{min}^{-1}\right)$ significantly reduced the centre oscillation velocity compared with the control-depleted cells $\left(1.68 \pm 0.06 \mu \mathrm{m} \mathrm{min}^{-1}\right)$. The centromeres in the NuSAP- or Kid-depleted cells had a lower proportion in the large velocity range $\left(2-4 \mu \mathrm{m} \mathrm{min}^{-1}\right)$ than that in the control cells (Fig. 3f), indicating that the depletion of NuSAP or Kid markedly reduced the high velocity of oscillation. As the velocity varies with the position of the centromere during oscillation and the maximum velocity is reached near the equator with the minimum $\operatorname{ICD}^{7,38}$, we further investigated whether 
a

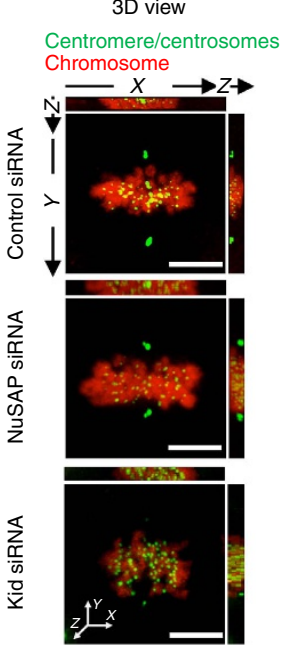

b Centromere track
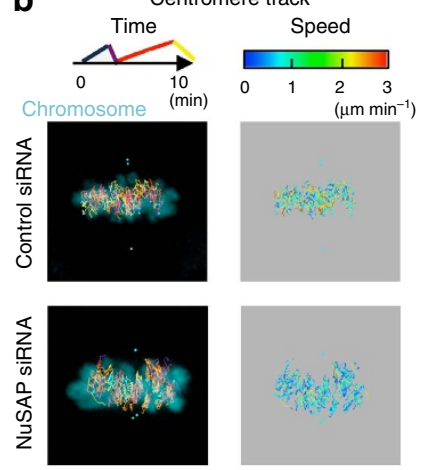

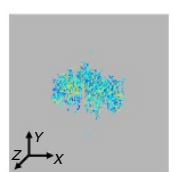

d Control siRnA
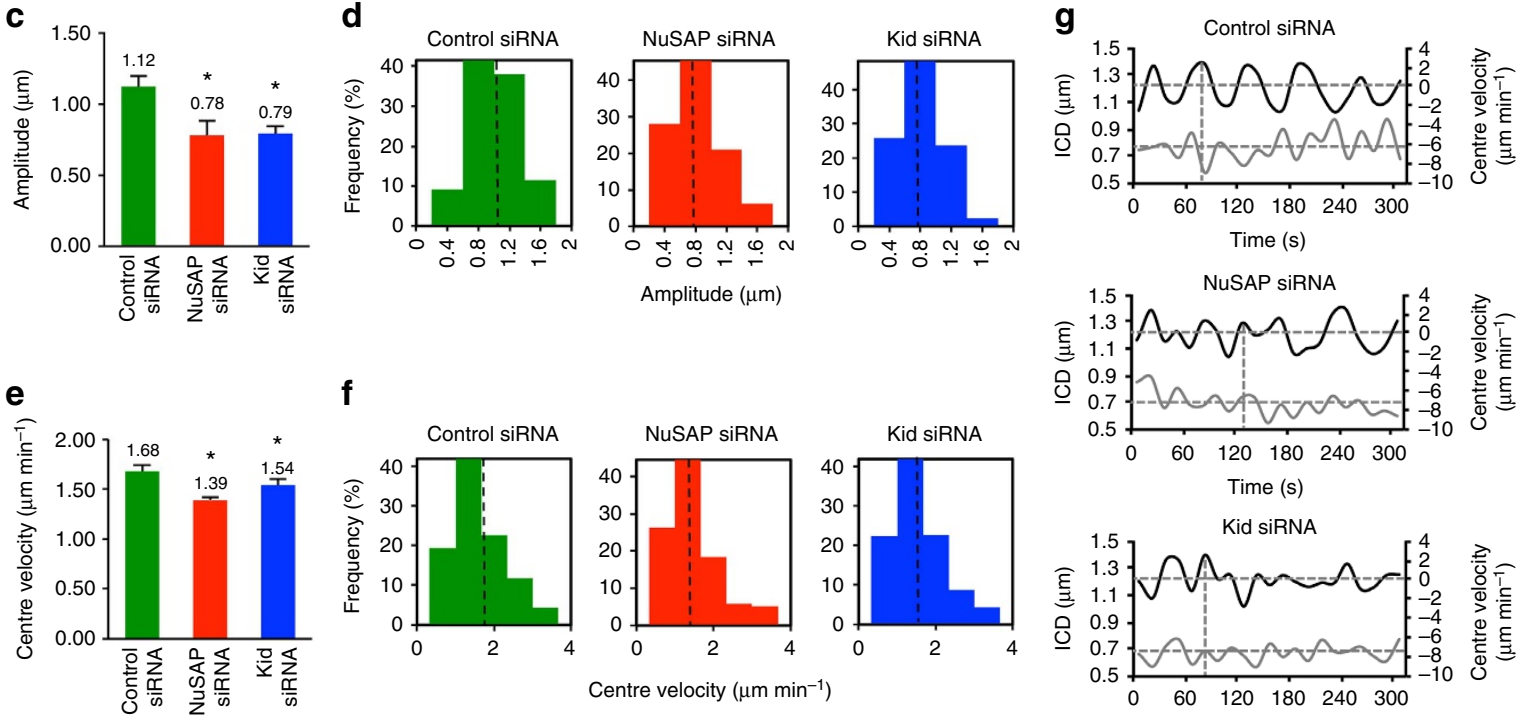

f
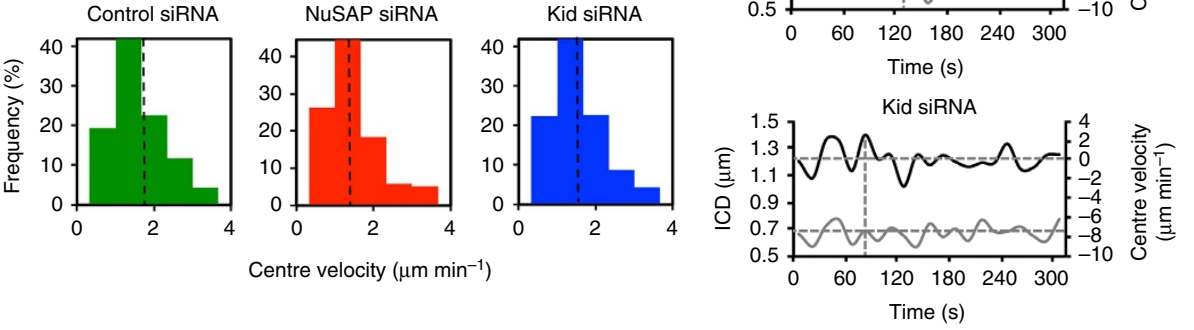

Figure 3 | The depletion of NuSAP or Kid attenuates the amplitude and velocity of centromere movements. (a) Representative images of the $3 D$ view using $x z$ and $y z$ projections in the control, NuSAP- or Kid-depleted synchronized metaphase mCherry-H2B HeLa cells. Centromeres were marked with GFP-CENPA and centrosomes with GFP-centrin. Scale bar, $5 \mu \mathrm{m}$. (b) Representative images of 3D centromere tracks, colour-coded for time and velocity. (c,d) Bar chart and histogram representing the average (c) and the distribution (d) of the amplitude of centromere oscillation in the control, NuSAP- or Kid-depleted metaphase cells. Error bars represent + s.e.m. ${ }^{\star} P<0.001$, by $t$-test. (e,f) Bar chart and the histogram representing the average (e) and the distribution (f) of the centre velocity. Error bars represent + s.e.m. ${ }^{\star} P<0.001$, by $t$-test. (g) Representative velocity (solid black line) and ICD (solid grey line) versus time plots of the projected centre in a sister centromere pair in control, NuSAP- and Kid-depleted metaphase cells. The sign of the velocity indicates the direction of the centromere movement. The average of the ICD (horizontal) and the minimum (horizontal) and maximum velocities (vertical) are indicated by dashed lines.

NuSAP and Kid can regulate the coupling of the velocity and the ICD versus time and position. As shown in Fig. 3g, in control cells, the maximum velocity was reached near the minimum ICD with approximately twice the frequency, consistent with a previous study ${ }^{38}$. However, in NuSAP- or Kid-depleted cells, the change in velocity was considerably irregular and coupling between velocity and ICD was abolished (Fig. 3g, rows 2 and 3). The function of NuSAP on chromosome oscillation was further confirmed using NuSAP siRNA (Supplementary Fig. 3a-c), and to rule out a possible dominant-negative effect, rescue experiments using mCherry-NuSAP expression in NuSAP siRNA-depleted cells were also conducted (Supplementary Fig. 3b,c). Overexpression of mCherry-NuSAP, but not mCherry vector, facilitated KT oscillation in NuSAP-depleted cells. Taken together, the data suggest that depletion of NuSAP or
Kid attenuates the overall motility and coupling of centromere oscillation.

NuSAP and Kid regulate centromere movement at iMTs. Our findings indicate that NuSAP and Kid tune the amplitude and velocity of centromere oscillation. However, we could not rule out the possibility that the regulation of chromosome movement by NuSAP may also result from its influence on kMTs. To examine this possibility, we depleted Nuf2 using siRNA to specifically diminish kMTs in the cells ${ }^{39}$, and subsequently characterized centromere oscillation in NuSAP/Nuf2 or Kid/Nuf2 double-depleted cells using Nuf2-depleted cells as a control (Supplementary Fig. 4b). The depletion of Nuf2 was further examined by immunofluorescence, which showed that KT-microtubule attachment was damaged in Nuf2-depleted 

Table 1 I Summary of the parameters defining centromere oscillation in bipolar and monopolar control, NuSAP, Kid, Nuf2,
Nuf2/NuSAP and Nuf2/Kid siRNA cells.

\begin{tabular}{|c|c|c|c|c|c|}
\hline SIRNA & Centre velocity $\left(\mu \mathrm{m} \min ^{-1}\right)$ & Amplitude $(\mu \mathrm{m})$ & Half-period (s) & ICD $(\mu \mathrm{m})$ & No. of KT pairs/no. of cells \\
\hline \multicolumn{6}{|l|}{ Bipolar } \\
\hline NuSAP & $v_{2}=1.39 \pm 0.03$ & $0.78 \pm 0.11$ & $32.56 \pm 3.55$ & $0.69 \pm 0.09$ & $226 / 8$ \\
\hline Nuf2 & $v_{4}=1.73 \pm 0.06$ & $1.15 \pm 0.08$ & $33.48 \pm 6.55$ & $0.61 \pm 0.13$ & $467 / 15$ \\
\hline NuSAP/Nuf2 & $v_{5}=1.46 \pm 0.03$ & $0.92 \pm 0.06$ & $33.24 \pm 2.93$ & $0.60 \pm 0.08$ & $257 / 11$ \\
\hline Kid/Nuf2 & $v_{6}=1.59 \pm 0.04$ & $0.95 \pm 0.06$ & $33.34 \pm 2.89$ & $0.58 \pm 0.10$ & $238 / 9$ \\
\hline Control $2^{\dagger}$ & $v_{13}=1.70 \pm 0.11$ & $1.18 \pm 0.12$ & $33.83 \pm 4.36$ & $0.73 \pm 0.08$ & $263 / 9$ \\
\hline \multicolumn{6}{|l|}{ Monopolar } \\
\hline Control1 & $v_{7}=1.39 \pm 0.10$ & $0.69 \pm 0.04$ & $31.06 \pm 2.88$ & $0.57 \pm 0.11$ & $358 / 15$ \\
\hline NuSAP & $v_{8}=1.25 \pm 0.06$ & $0.66 \pm 0.04$ & $30.65 \pm 2.87$ & $0.56 \pm 0.07$ & $261 / 10$ \\
\hline Kid & $v_{9}=0.76 \pm 0.02$ & $0.49 \pm 0.03$ & $28.66 \pm 2.22$ & $0.52 \pm 0.08$ & $447 / 13$ \\
\hline Nuf2 & $v_{10}=1.58 \pm 0.09$ & $0.83 \pm 0.05$ & $29.97 \pm 3.04$ & $0.52 \pm 0.09$ & $341 / 15$ \\
\hline NuSAP/Nuf2 & $v_{11}=1.03 \pm 0.03$ & $0.56 \pm 0.05$ & $26.96 \pm 2.17$ & $0.47 \pm 0.08$ & 237/11 \\
\hline
\end{tabular}

cells (Supplementary Fig. 4c). The depletion of Nuf2 resulted in $\sim 84 \%$ of cells with misaligned chromosomes (Supplementary Fig. 4d), which is consistent with previous studies ${ }^{36,40,41}$. Our result indicates that Nuf2 was strongly depleted, removing most KT fibres; however, since it was not completely depleted, the residual kMTs might still be present. Representative 3D timelapse images are shown in Fig. 4a and Supplementary Movie 3, and the centromere tracks, colour-coded for time, are shown in Fig. $4 \mathrm{~b}$, lane 1 . The movement of centromeres in Nuf2-depleted cells displayed a faster velocity with more tracks shown in yellowred $\left(2-3 \mu \mathrm{m} \mathrm{min}^{-1}\right)$ when colour-coded for velocity (Fig. $4 \mathrm{~b}$, lane 2, top). On the other hand, the velocities of centromeres in the NuSAP/Nuf2 and Kid/Nuf2 double-depleted cells were mostly in the $0-2 \mu \mathrm{m} \mathrm{min}^{-1}$ range (shown as blue and green in Fig. $4 \mathrm{~b}$, lane 2, middle and bottom), indicating that the velocity of centromere movement was dramatically decreased by co-depletion of NuSAP or Kid in Nuf2-depleted cells.

The amplitude of the centre oscillation in NuSAP/Nuf2- or Kid/Nuf2-depleted cells was dramatically reduced compared with that in Nuf2-depleted cells (Fig. 4c). In addition, the frequency of oscillations with high amplitude $(1-2 \mu \mathrm{m})$ was significantly decreased by co-depletion with NuSAP or Kid in the Nuf2-depleted cells (Fig. 4d). The velocity of the centre was also significantly decreased in the NuSAP/Nuf2- and Kid/Nuf2depleted cells (Fig. 4e) and showed a largely decreased proportion in the high velocity range, compared with that in Nuf2-depleted cells $\left(2-4 \mu \mathrm{m} \mathrm{min}^{-1}\right.$, Fig. $\left.4 \mathrm{f}\right)$. The coupling of the maximum velocity with the minimum ICD was also abolished in Nuf2-, NuSAP/Nuf2- and Kid/Nuf2-depleted cells (Fig. 4g, vertical dotted lines), indicating that this linkage requires kMT attachment. The overall attenuation of oscillation in the absence of kMTs following depletion of Nuf2 suggests that the elimination of chromosome oscillation by NuSAP or Kid is predominantly on iMTs.

NuSAP and Kid enhance KT oscillation in monopolar cells. In bipolar cells, centromere oscillation is regulated by the motors, the interpolar and kMTs from two poles with opposite directions. To simplify the factors involved in chromosome oscillation further, we studied the movement of centromeres in monastroltreated monopolar cells with both iMTs and kMTs from one spindle pole with a single direction ${ }^{2,4,10,38}$. Representative 3D time-lapse images (Fig. 5a) and colour-coded centromere tracks (Fig. 5b, lane 1) of the control, NuSAP- or Kid-depleted cells are shown in Fig. 5 (Supplementary Movie 4). The centromere tracks indicate that centromere movement in NuSAP-depleted or Kid-depleted cells was slower, with more tracks shown in blue (0-1 $\left.\mu \mathrm{m} \mathrm{min}^{-1}\right)$ compared with the control cells (Fig. 5b, lane 2).

Depletion of NuSAP or Kid in monopolar cells resulted in an even more severe decrease in oscillation amplitude (Fig. $5 \mathrm{c}$ ), with a greater proportion in the $0-1 \mu \mathrm{m}$ range compared with the control monopolar cells (Fig. 5d). Furthermore, the velocity of the centre was significantly decreased in NuSAP- or Kid-depleted monopolar cells, with a dramatically decreased proportion in the range of $2-4 \mu \mathrm{m} \mathrm{min}^{-1}$ (Fig. 5e,f). We noted that the effect of Kid depletion on oscillation amplitude and velocity in monopolar cells was greater than that of NuSAP (Fig. 5e,f, lane 3). Because the PEF generated by Kid is directly related to the density of the iMTs at the function positions ${ }^{12,37}$, the higher density of microtubules in the monopolar aster structure compared with the bipolar spindle might result in an increase in the Kid-generated PEF. This is consistent with a previous finding that mono-oriented chromosome arms are immediately ejected outwards once cut free ${ }^{4}$. Moreover, the depletion of NuSAP may affect both the PF at kMTs and the PEF at iMTs with opposing directions, leading to a reduced overall effect on chromosome movement. The coupling of velocity and ICD was abolished in the control, NuSAP- and Kid-depleted monopolar cells (Fig. 5g), indicating that non-random centromere oscillation also requires a bipolar spindle structure. Taken together, these data suggest that both NuSAP and Kid positively affect the amplitude and velocity of centromere oscillation in monopolar cells.

NuSAP tunes the PEF with Kid at iMTs in monopolar cells. Using experiments combining both the depletion of Nuf2 to disrupt kMTs, and monastrol treatment to form monopolar spindles, we specifically investigated the PEF on iMTs with a single direction in the Nuf2-, NuSAP/Nuf2- or Kid/Nuf2-depleted 
a

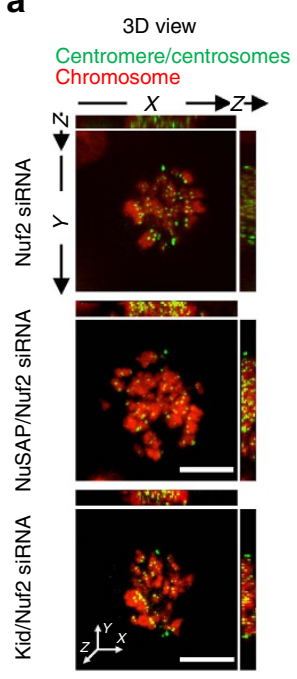

b

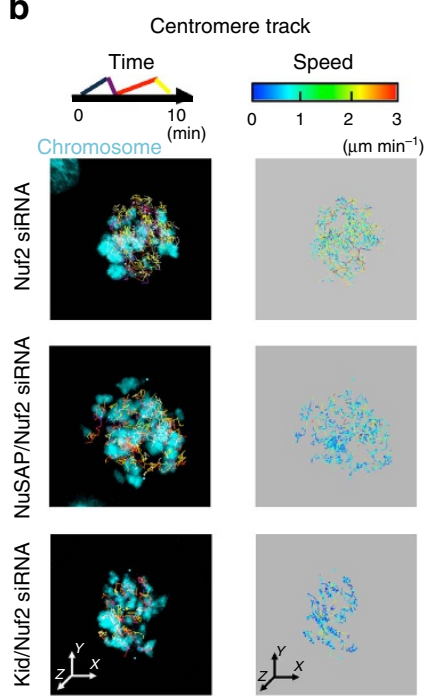

C

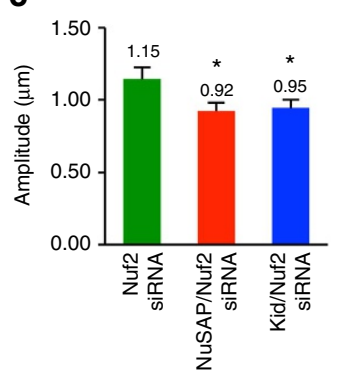

e

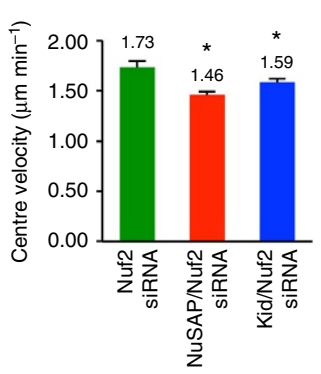

d

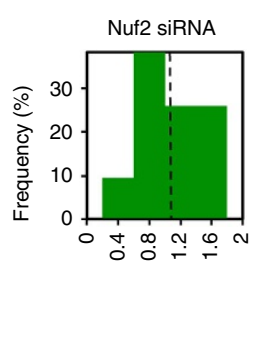

f

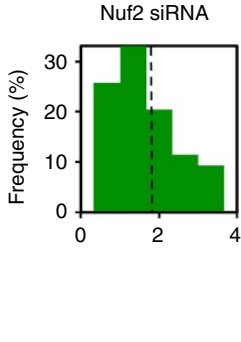

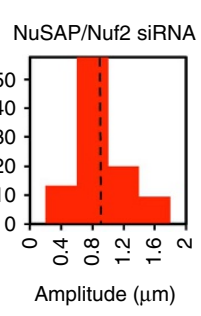

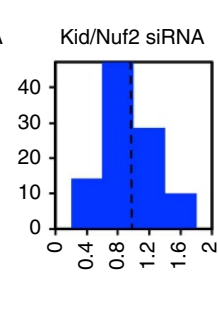

g
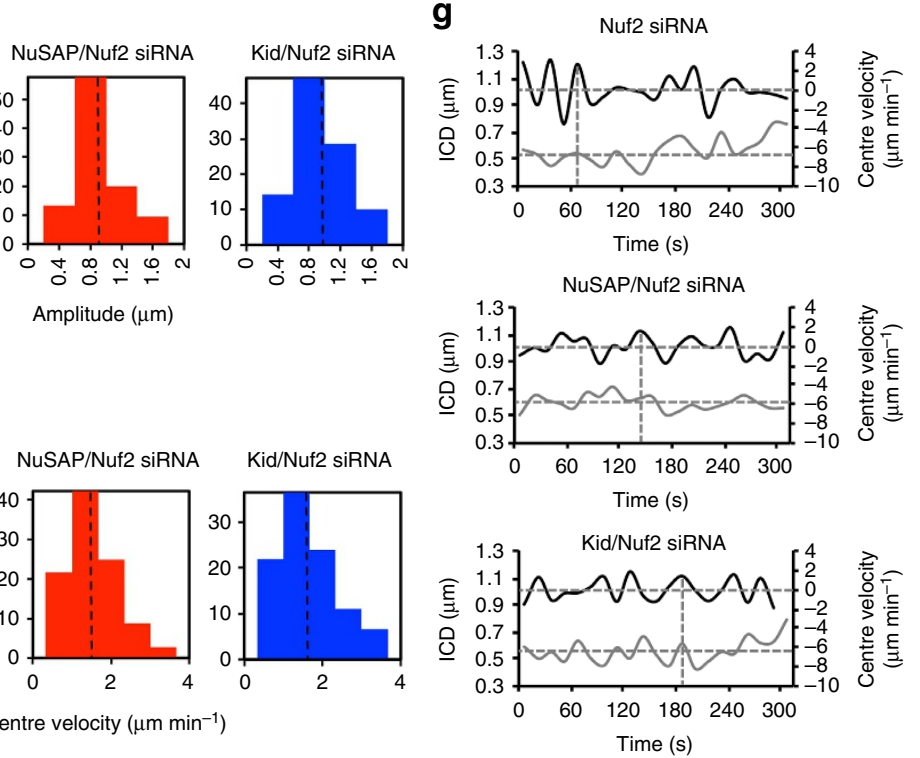

Figure 4 | NuSAP and Kid regulate centromere movement at interpolar microtubules. (a) Representative images of the 3D view using $x z$ and $y z$ projections in Nuf2-, NuSAP/Nuf2- or Kid/Nuf2-depleted synchronized metaphase HeLa cells stably expressing mCherry-H2B. Centromeres were marked with GFP-CENPA and centrosomes with GFP-centrin. Scale bar, $5 \mu \mathrm{m}$. (b) Representative images of the 3D centromere tracks colour-coded for time and velocity. (c,d) Bar chart and histogram representing the average (c) and the distribution (d) of the amplitude of centromere oscillation in Nuf2-, NuSAP/Nuf2- or Kid/Nuf2-depleted metaphase cells. Error bars represent + s.e.m. ${ }^{\star} P<0.001$, by $t$-test. (e,f) Bar chart and histogram representing the average (e) and the distribution (f) of the centre velocity. Error bars represent + s.e.m. ${ }^{\star} P<0.001$ by $t$-test. ( $(\mathbf{g})$ Representative velocity (black line) and ICD (grey line) versus time plots of the projected centre in a sister centromere pair in Nuf2-, NuSAP/Nuf2- or Kid/Nuf2-depleted metaphase cells. The sign of the velocity indicates the direction of centromere movement. The average of the ICD (horizontal) and the minimum (horizontal) and maximum velocities (vertical) are indicated by the dashed lines.

monopolar cells. The representative 3D time-lapse live-cell images and centromere tracks (Fig. 6a,b and Supplementary Movie 5) show that oscillation was dramatically retarded with a significant decrease in velocity in the NuSAP/Nuf2- and Kid/Nuf2-depleted cells (Fig. 6b, lane 2, middle and bottom).

The amplitude of oscillation at the centre point in NuSAP/ Nuf2 $(0.56 \mu \mathrm{m})$ - and Kid/Nuf2 $(0.66 \mu \mathrm{m})$-depleted monopolar cells was also significantly reduced compared with that in control cells $(0.83 \mu \mathrm{m})$, as shown by the substantial decrease in both the average amplitude (Fig. 6c) and the proportion in the $1-2 \mu \mathrm{m}$ range (Fig. 6d). Moreover, the centre velocity was significantly attenuated in NuSAP/Nuf2 $\left(1.03 \mu \mathrm{m} \mathrm{min}^{-1}\right)$ - and Kid/Nuf2 $\left(1.21 \mu \mathrm{m} \mathrm{min}^{-1}\right)$-depleted monopolar cells compared with that in control cells $\left(1.58 \mu \mathrm{m} \mathrm{min}^{-1}\right.$; Fig. 6e,f). The significant decrease in oscillation velocity and amplitude in both the NuSAP/Nuf2- and Kid/Nuf2-depleted cells suggests that NuSAP strongly regulates the forces on iMTs, particularly the PEF generated by Kid. Compared with the depletion of Kid/Nuf2, co-depletion of NuSAP and Nuf2 had a greater effect on chromosome oscillation (Fig. 6e,f, lane 2). This indicates that NuSAP might also facilitate other PEFs at iMTs besides that generated by Kid. The linkage between velocity and ICD during oscillation was also dramatically reduced in Nuf2-, NuSAP/Nuf2and Kid/Nuf2-depleted monopolar cells, with extremely random movements observed (Fig. 6g; vertical dotted lines). This is consistent with our previous results (Figs 3-5g) showing that 
a

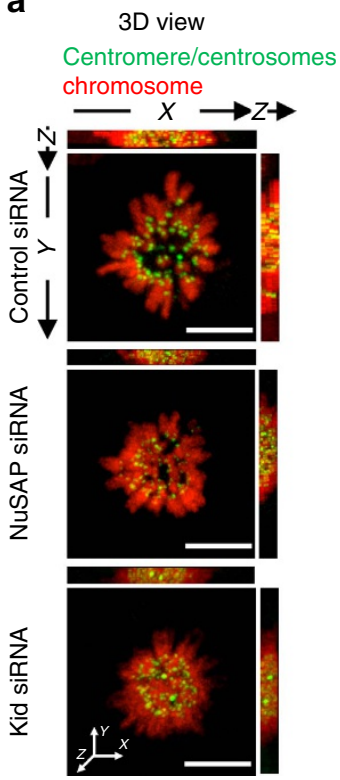

b

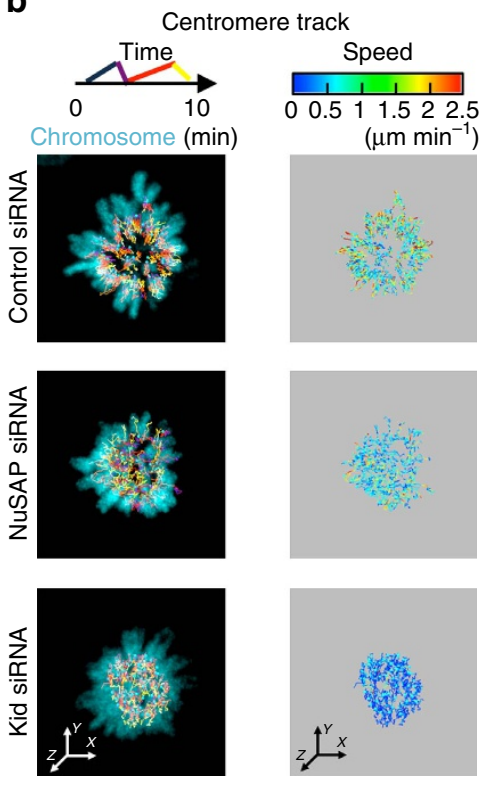

C

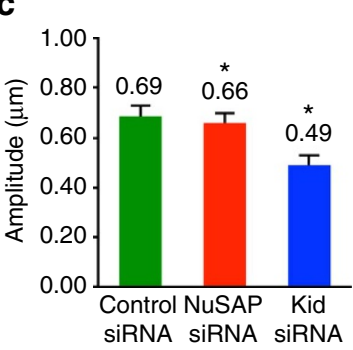

e

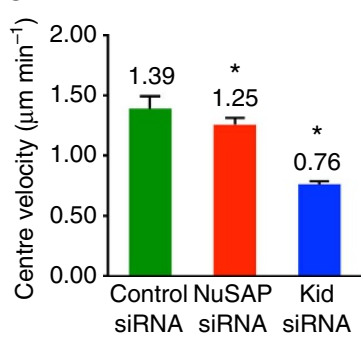

d

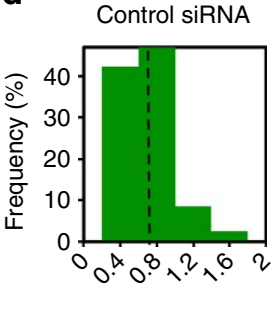

NuSAP SiRNA

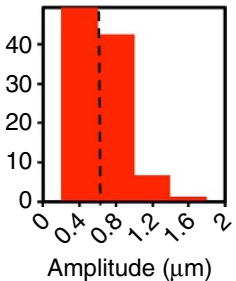

f

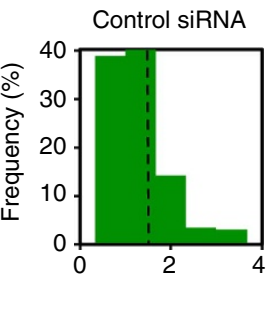

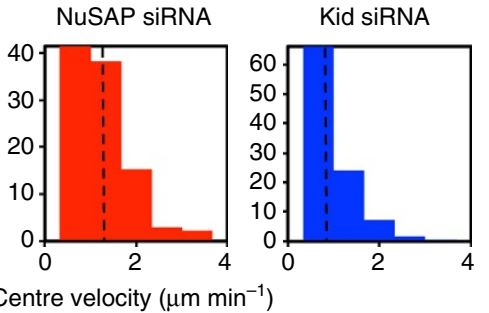

9
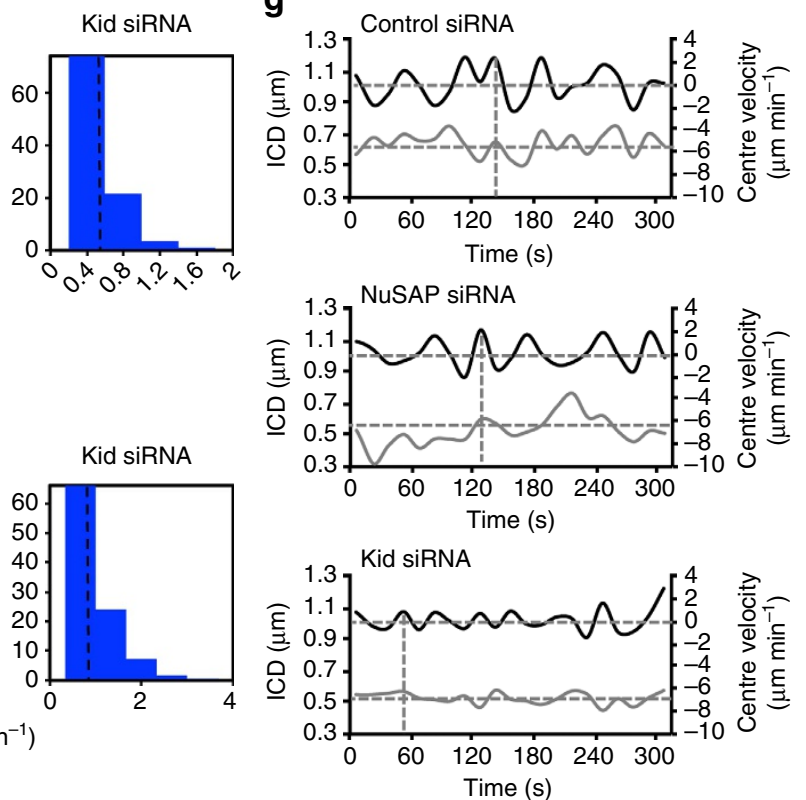

Figure 5 | The effects of NuSAP and Kid depletion on the amplitude and velocity of centromere oscillation in monopolar cells are correlated.

(a) Representative images of the 3D view using $x z$ and $y z$ projections in the control, NuSAP- or Kid-depleted monastrol-treated monopolar HeLa cells stably expressing mCherry-H2B. Centromeres were marked with GFP-CENPA and centrosomes with GFP-centrin. Scale bar, $5 \mu \mathrm{m}$. (b) Representative images of the $3 \mathrm{D}$ centromere tracks colour-coded for time and velocity. (c,d) Bar chart and histogram representing the average (c) and the distribution (d) of the amplitude of centromere oscillation in the control, NuSAP- or Kid-depleted monopolar cells. Error bars represent + s.e.m. ${ }^{\star} P<0.001$, by $t$-test. $(\mathbf{e}, \mathbf{f})$ Bar chart and histogram representing the average (e) and the distribution (f) of the centre velocity. Error bars represent + s.e.m. ${ }^{\star} P<0.001$, by $t$-test. (g) Representative velocity (black line) and ICD (grey line) versus time plots of the projected centre in a sister centromere pair in the control, NuSAP- or Kid-depleted monopolar cells. The sign of the velocity indicates the direction of centromere movement. The average of the ICD (horizontal) and the minimum (horizontal) and maximum velocities (vertical) are indicated by the dashed lines.

kMT attachment and a bipolar spindle structure are both required for proper chromosome oscillation. Taken together, these data suggest that NuSAP governs chromosome oscillation by strongly tuning the Kid-generated PEF on iMTs.

NuSAP governs the PEF generated by Kid during KT oscillation. To validate our statistical analysis biologically, we conducted a microtubule gliding assay using purified Kid and NuSAP proteins
(Supplementary Fig. 6a). As the kymographs shown in Fig. 7a,b, microtubules glided on the Kid-coated surface slowly. Kid was found to be a slow motile motor with a speed of $27.8 \pm 2.6 \mathrm{~nm} \mathrm{~s}^{-1}$, compared with the speed of K560 $\left(311 \pm 23 \mathrm{~nm} \mathrm{~s}^{-1}\right.$, Supplementary Fig. 6b). However, in the presence of NuSAP protein, the gliding speed was significantly enhanced $\left(46.5 \pm 9.1 \mathrm{~nm} \mathrm{~s}^{-1}\right.$; Fig. $7 \mathrm{c}$ and Supplementary Movie 6). These results indicate an important role of NuSAP on facilitating Kid motility in vitro, which further supports its role 
a

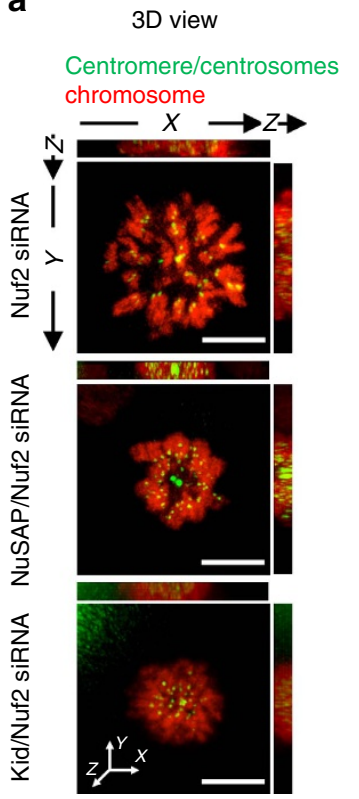

b

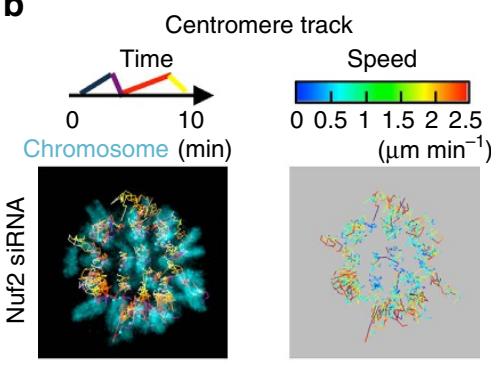

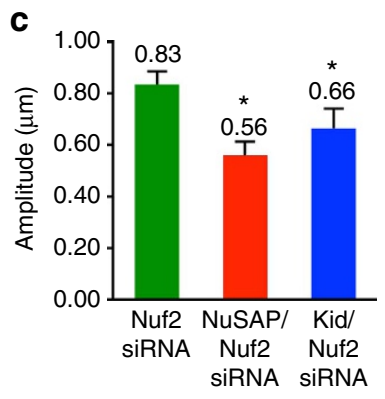

e

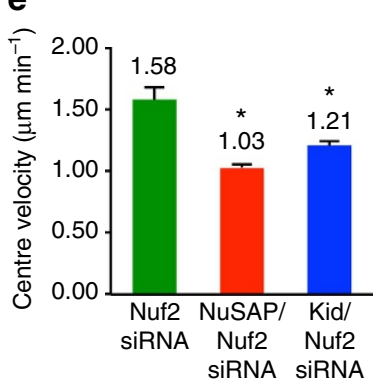

d
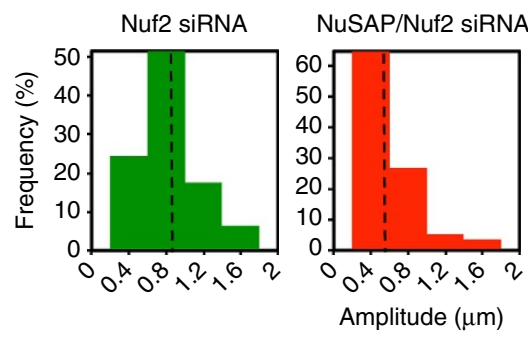

f

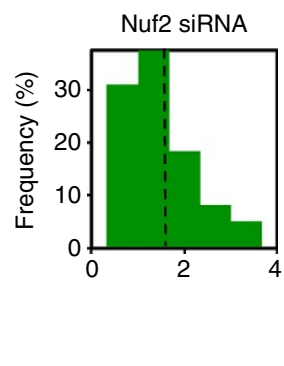

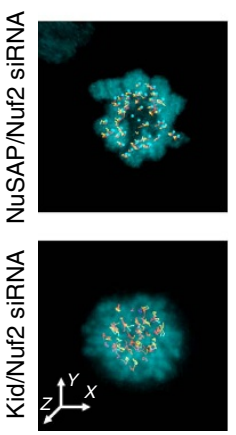
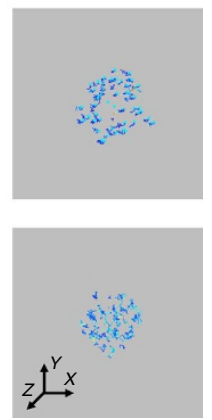

g
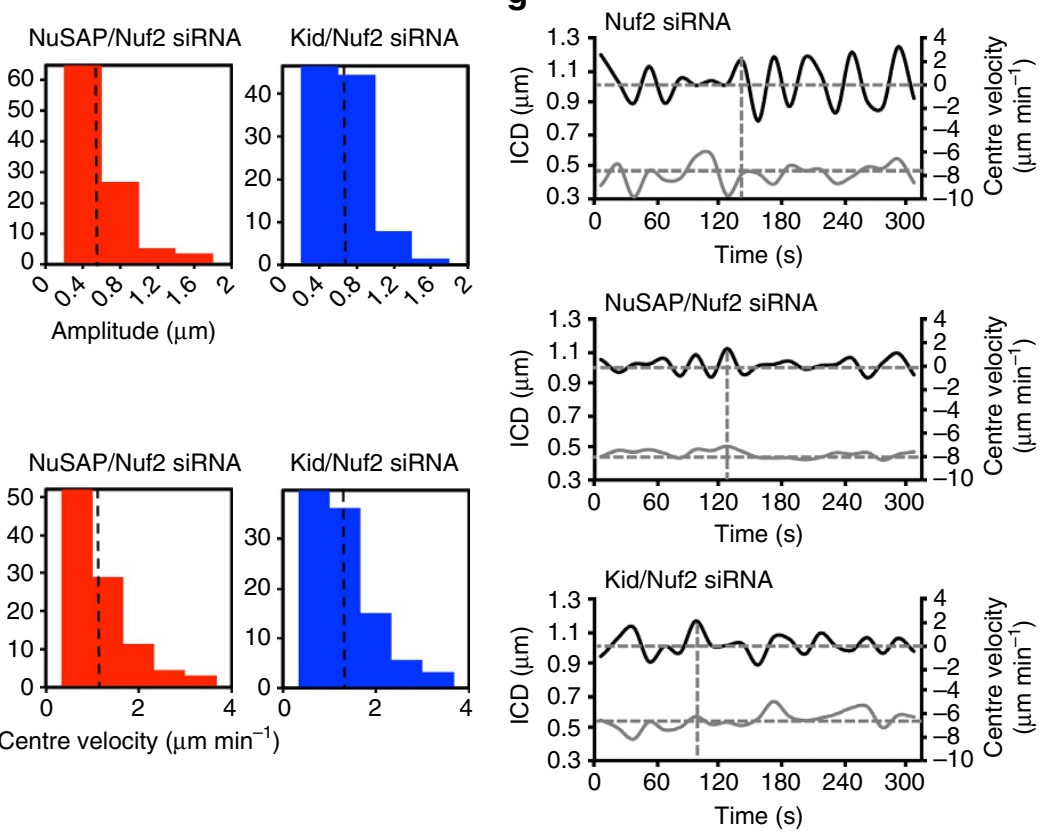

Figure 6 | NuSAP tunes the polar ejection force with Kid at interpolar microtubules in monopolar cells. (a) Representative images of the 3D view using $x z$ and $y z$ projections in Nuf2-, NuSAP/Nuf2- or Kid/Nuf2-depleted monastrol-treated monopolar HeLa cells stably expressing mCherry-H2B. Centromeres were marked with GFP-CENPA and centrosomes with GFP-centrin. Scale bar, $5 \mu \mathrm{m}$. (b) Representative images of 3D centromere tracks colour-coded for time and velocity. (c,d) Bar chart and histogram representing the average (c) and the distribution (d) of the amplitude of centromere oscillation in Nuf2-, NuSAP/Nuf2- or Kid/Nuf2-depleted monopolar cells. Error bars represent + s.e.m. ${ }^{\star} P<0.001$, by $t$-test. (e,f) Bar chart and histogram representing the average (e) and the distribution (f) of the centre velocity. Error bars represent + s.e.m. ${ }^{\star} P<0.001$, by $t$-test. ( $\mathbf{g}$ ) Representative velocity (black line) and ICD (grey line) versus time plots of the projected centre in a sister centromere pair in the control, NuSAP- or Kid-depleted monopolar cells. The sign of the velocity indicates the direction of centromere movement. The average of the ICD (horizontal) and the minimum (horizontal) and maximum velocities (vertical) are indicated by the dashed lines.

on Kid-generated PEF during chromosome oscillation in metaphase cells. To further confirm the regulation of NuSAP on Kid-generated PEF in cells, a double depletion of both NuSAP and Kid was conducted in both bipolar and monopolar cells (Supplementary Fig. 6c,d). Our results show that double depletion of NuSAP and Kid resulted in a severe decrease in oscillation amplitude and the velocity of the centre in both metaphase and monopolar cells (Fig. 7d,e). The chromosome behaviour in the double depletion of NuSAP and Kid cells is similar to that in the depletion of NuSAP or Kid cells (Figs 3-6c,e), which proves that the function of NuSAP on the PEF conducted by Kid is critical for its role in chromosome oscillation in cells.

Our study also indicates a possible role of NuSAP on kMTs. Previous studies have indicated that NuSAP can stabilize microtubules and link them to chromosomes ${ }^{14-16}$. Thus, NuSAP may facilitate the PF generated by kMTs through its 
regulation of microtubule dynamics. To separate the role of NuSAP on kMTs, the cells were cold-treated. Cold-resistant kMTs in NuSAP-depleted cells were dramatically decreased, whereas a greater abundance of kMTs was observed in GFPNuSAP-overexpressing cells (Fig. 7f). To better address this question, an fluorescence dissipation after photoactivation assay was performed (Fig. 7g). The KT region around the metaphase plate was photoactivated in PAGFP $\alpha$-tubulin-expressing cells transfected with control siRNA, NuSAP siRNA, mCherry or mCherry-NuSAP, and the half-lives of kMTs were analysed with a double exponential equation ${ }^{42,43}$ (Fig. $7 \mathrm{~h}$ ). The increased halflives of kMTs in NuSAP-overexpressed cells indicate that NuSAP functions as a kMT stabilizer in mitotic spindles. The effects of NuSAP on kMTs and iMTs were further deciphered using FRAP a

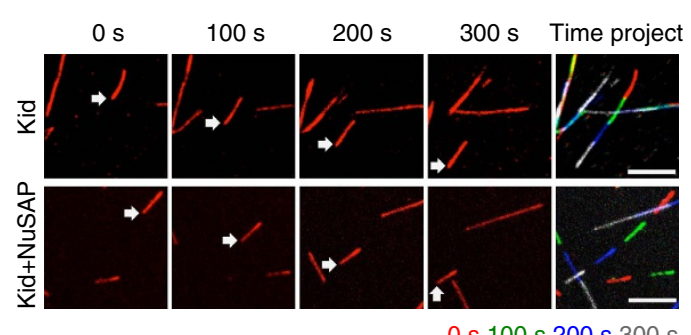

d

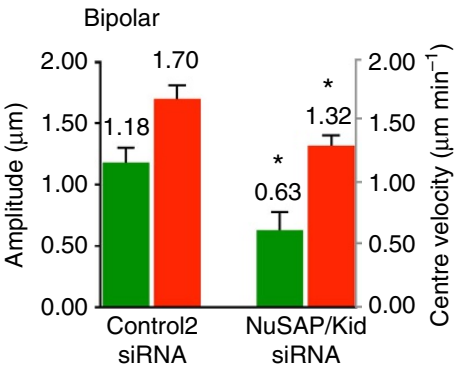

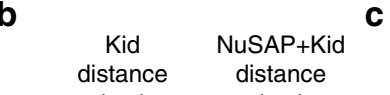
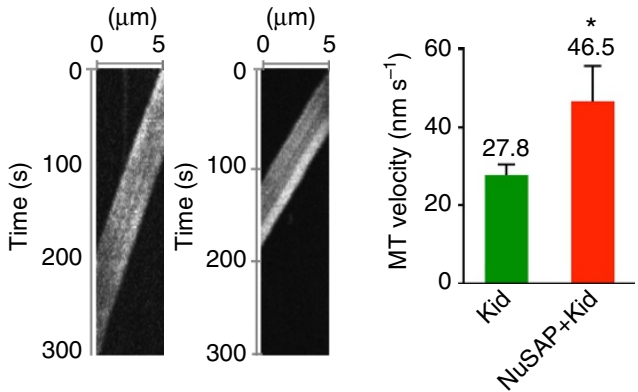

e

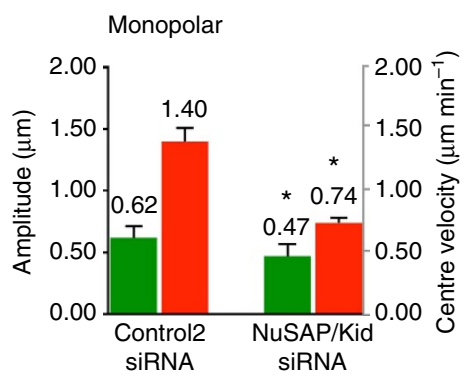

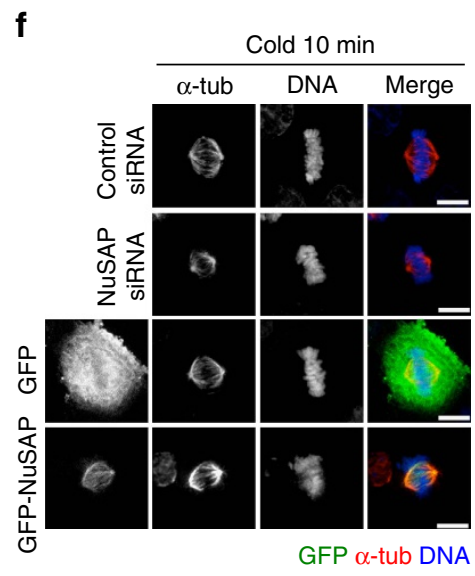

g

h

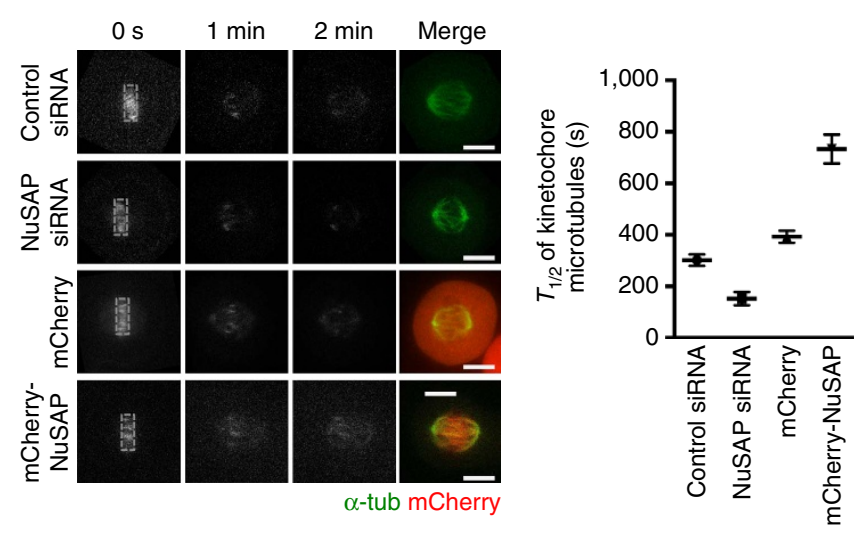

i

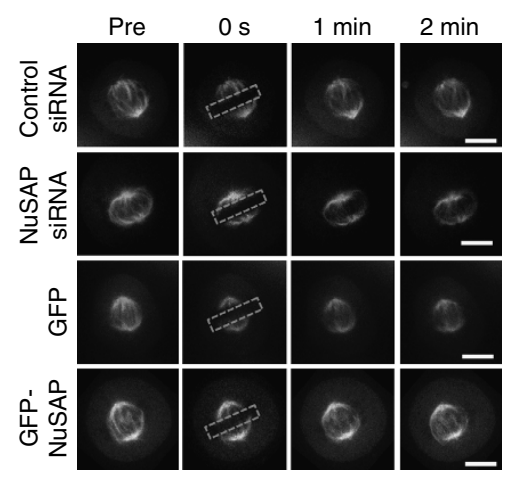

j

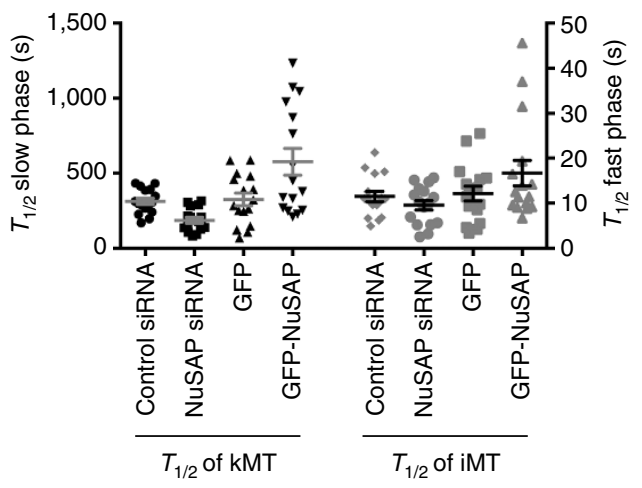


(fluorescence recovery after photobleaching) assays (Fig. 7i,j). The microtubule signal in the KT region was photobleached and the fluorescence intensity decay within the region was analysed. As shown in Fig. 7j, NuSAP regulates the stability of both kMTs and iMTs; however, the half-lives of the kMTs were more dependent on NuSAP compared with the half-lives of the iMTs. Our results indicate a function of NuSAP on kMT stability, which may explain its role on kMT-generated PF during chromosome oscillation in metaphase.

\section{Discussion}

Here we show that the MAP, NuSAP, plays an important role in chromosome congression at metaphase. Previous studies have indicated that NuSAP is a microtubule stabilizer during mitosis, both in vitro and in vivo ${ }^{14-16}$. Our results extend these earlier findings by showing that NuSAP associates with the central spindle microtubules to regulate the alignment and orientation of the chromosome at the metaphase plate, facilitating proper chromosome congression during metaphase.

We further identify NuSAP as a novel regulator of the chromokinesin Kid. NuSAP associates with Kid to facilitate its distribution on microtubules. NuSAP and Kid are both expressed at high levels during mitosis ${ }^{19,44}$, and are regulated by the RanGTP gradient to accumulate at the metaphase plate ${ }^{15,45}$. We demonstrate that NuSAP binds Kid and plays a pivotal role in regulating Kid-specific functions during metaphase, and that this interaction facilitates the association of Kid with microtubules. The temporal and spatial nature of the regulation indicates that NuSAP actively regulates Kid during metaphase.

Kid functions as a key force-producing agent of the $\mathrm{PEF}^{30,46}$, and our quantitative live-cell imaging experiments show that NuSAP specifically regulates chromosome oscillation by governing the PEF generated by Kid. The effects of NuSAP depletion on the regulation of the amplitude and velocity of chromosome oscillation in both bipolar and monopolar cells correlate with those resulting from Kid depletion. The synergistic regulation of centromere movement by NuSAP with Kid occurs specifically at iMTs, as shown in the absence of kMTs following Nuf2 depletion. Our results suggest that the PEF produced by Kid depends significantly on the specific regulation by NuSAP. This is further supported by microtubule gliding assays using purified Kid and NuSAP proteins, which prove that full-length Kid is a motile motor with a slow speed $\left(27.8 \mathrm{~nm} \mathrm{~s}^{-1}\right)$. NuSAP positively facilitates microtubule gliding on Kid-coated surfaces, which further supports our analysis that NuSAP is important in the regulation of the Kid-generated PEF. Furthermore, to confirm our analysis in cell-based experiments, the double depletion of NuSAP and Kid was conducted and the results further evidence the major role of NuSAP on the Kid-generated PEF both in cells and in vitro. The differences between the double depletion of NuSAP/Kid from the depletion of NuSAP or Kid suggest other possible important functions of NuSAP on chromosome oscillation besides its regulation on Kid. Our results also indicate that NuSAP regulates the stability of iMTs, which might further influence the force generated by the dynamics of iMTs ${ }^{37}$. Other kinesins, for example, Kif4a (refs 7,8) and dyneins ${ }^{47}$, involved in chromosome oscillation may be also regulated by NuSAP, and it would be interesting to study how other forces are influenced by NuSAP.

Our analysis also indicates that NuSAP has a moderate effect on the regulation of kMTs. We further examined the role of NuSAP in spindle microtubule stability and confirmed the function of NuSAP on both interpolar and kMT stability. The stability of kMTs is important for their ability to generate the PF. Thus, NuSAP may regulate the kMT-generated PF through its regulation on kMT stability. Our finding suggests that NuSAP may also influence the kMT-generated PF through the regulation of other motors on kMTs. Several other microtubule motors that play important roles in kMT dynamics and KT attachment may be involved in the PF generated by kMTs, for example, MCAK, which functions as a microtubule depolymerizer to regulate chromosome alignment and KT attachment ${ }^{48-51}$, and Kif18a, a microtubule plus-end-directed motor with depolymerase activity that has been implicated in proper chromosome oscillation $^{6,52}$

Using a simplified chromosome centre-projected approach, our data suggest that the average ICD and half-period did not change significantly in NuSAP- or Kid-depleted cells. However, the distribution of the ICD and half-period were influenced, and coupling of the ICD and velocity was abolished by the depletion of NuSAP or Kid. However, the complicated nature of chromosome oscillation means that it is possible that other factors might also be involved. For example, the stiffness of KTs absolves one portion of the unbalanced force on the chromosome during oscillation ${ }^{9,11,36,53}$, and changes in microtubule density in the absence of kMTs or in monopolar MT asters may also lead to changes in the $\mathrm{PEF}^{2,4,9,37}$. Further studies are needed to answer these questions.

In summary, our results show that NuSAP facilitates chromosome congression and interacts with Kid to regulate its localization during metaphase. Thus, NuSAP regulates chromosome oscillation through its regulations on both the stability of the kMTs and the Kid-generated PEF during metaphase.

Figure 7 | NuSAP governs Kid-generated PEF during chromosome oscillation. (a) Representative images and colour project of MT gliding assay with Kid or NuSAP and Kid proteins. Arrows represent one MT gliding at different time points. Images were acquired in a $2 \mathrm{~s}$ interval for 5 min. Scale bar, $3 \mu \mathrm{m}$. (b) Kymographs showing a sample microtubule gliding on Kid-coated surfaces in the presence or absence of NuSAP protein. (c) Bar chart representing the average velocity of MT gliding on Kid-coated surfaces in the presence or absence of NuSAP protein. Error bars represent $\pm s$.d. ${ }^{\star} P<0.01$, by $t$-test. (d) Bar chart representing the average of the amplitude (black) and centre velocity (grey) of centromere oscillation in control- or NuSAP/Kid-doubledepleted metaphase cells. Error bars represent + s.e.m. ${ }^{\star} P<0.001$, by $t$-test. (e) Bar chart representing the average of the amplitude (black) and centre velocity (grey) of centromere oscillation in control- or NuSAP/Kid-double-depleted monopolar cells. Error bars represent + s.e.m. ${ }^{\star} P<0.001$, by $t$-test. (f) The kinetochore MTs in metaphase HeLa cells transfected with control siRNA, NuSAP siRNA, GFP-vector or GFP-NuSAP after cold treatment. Cells were stained with an anti- $\alpha$-tubulin antibody and DNA with Hoechst 33342. Scale bar, $5 \mu \mathrm{m}$. (g) Representative images of PAGFP $\alpha$-tubulin stability in metaphase HeLa cells transfected with control siRNA, NuSAP siRNA, mCherry vector or mCherry-NuSAP after photoactivation. Dotted squares represent photoactivated region. Scale bar, $5 \mu \mathrm{m}$. (h) Bar chart of the half-lives of spindle MTs calculated with a double exponential regression fitting of photoactivation assays. $n$ (control siRNA) $=14$ cells, $n($ NuSAP siRNA) $=13, n$ (mCherry) $=13, n$ (mCherry-NuSAP) $=12$. Error bars represent \pm s.d. (i) FRAP assays; representative images of MT dynamics in metaphase stable mCherry- $\alpha$-tubulin HeLa cells transfected with control siRNA, NuSAP siRNA, GFP-vector or GFP-NuSAP after photobleaching. Dotted squares represent the photobleaching region. Scale bar, $5 \mu \mathrm{m}$. (j) Dot plot representing the half-life of kinetochore microtubules (slow phase) and interpolar microtubules (fast phase) calculated according to a double exponential regression curve with FRAP assays in control siRNA-, NuSAP siRNA-, GFP-vector- or GFP-NuSAP-transfected metaphase cells. Mean \pm s.e.m. are indicated. 


\section{Methods}

Plasmids and siRNAs. The cDNAs of NuSAP, Kid and CENPA were amplified from a cDNA library extracted from HEK 293T cells and inserted into the pXJ40 vector tagged with GFP, mCherry, haemagglutinin (HA) or FLAG. His-NuSAP was inserted into the pET28b vector. GST-Kid was inserted into the pGEX-4T-1 vector. To construct Kid-GFP-His, Kid was first inserted into the EGFP-N1 vector and the Kid-GFP was then inserted into a modified pET32a vector with no other tags, which directly links Kid-GFP with the His tag followed by a termination codon. GFP-centrin1 was a gift from Dr Maki Murata-Hori. The mCherry $\alpha$-tubulin was obtained from Addgene (\#49149 (ref. 54)) and inserted into the pXJ40 vector tagged with GFP or HA. The K560-Dendra2 vector was a gift from Dr Jared C. Cochran. The sequences of NuSAP siRNA (5'-AAGCACCAAGAAGCTGAG AAT- $3^{\prime}$ (ref. 14)), NuSAP siRNA No.2 (ref. 21; Silencer Selected 27674, InvitrogenLife Technologies) and Nuf2 siRNA (5'-AAGCATGCCGTGAAACGTATA- ${ }^{\prime}$ (refs $36,39,41,55)$ ) were described previously and synthesized by Sigma. The siRNA oligonucleotide for Kid was synthesized by Santa Cruz Biotechnology (\#sc-44350) and Silencer Negative Control \#1 siRNA was used as a control (Ambion).

Cell culture and synchronization. HeLa and HEK 293T cells (American Type Culture Collection) were cultured in DMEM, supplemented with $10 \%$ fetal bovine serum (Gibco) and $1 \%$ penicillin/streptomycin at $37^{\circ} \mathrm{C}, 5 \% \mathrm{CO}_{2}$. Stable $\mathrm{H} 2 \mathrm{~B}-$ mCherry HeLa cells were generated using the pcDNA3-H2B-mCherry vector from Addgene (\#20972 (ref. 56)) and cultured in the presence of $0.2 \mathrm{mg} \mathrm{ml}^{-1} \mathrm{G} 418$ (Sigma). For DNA transfections, HEK 293T and HeLa cells were transfected using calcium phosphate and Effectene (Qiagen), respectively, to introduce target genes. For siRNA transfections, stable H2B-mCherry HeLa cells were transfected at 30$50 \%$ confluence, with $20 \mathrm{nM}$ of each siRNA using Lipofectamine 2000 (Invitrogen) according to the manufacturer's protocol. For experiments involving double depletions, control cells were treated with $40 \mathrm{nM}$ negative control siRNA. To synchronize cells at the G2/M stage, HeLa cells were treated with $100 \mathrm{ng} \mathrm{ml}^{-1}$ nocodazole (Sigma) for $16 \mathrm{~h}$, washed three times with $1 \times \mathrm{PBS}$ and released into DMEM medium containing $10 \mu \mathrm{M}$ MG132 (Sigma) for $2 \mathrm{~h}$ before fixation or liveimaging. For monopolar spindle experiments, cells were treated with $100 \mu \mathrm{M}$ monastrol (Sigma) for $2 \mathrm{~h}$ before fixation or live-imaging. To analyse the interaction between NuSAP and Kid during the cell cycle, HeLa cells were treated with $2 \mathrm{mM}$ thymidine (Sigma) for $24 \mathrm{~h}$, released for $3 \mathrm{~h}$ and treated with $100 \mathrm{ng} \mathrm{ml}^{-1}$ nocodazole for $11 \mathrm{~h}$, followed by $10 \mu \mathrm{M}$ MG132 treatment for $2 \mathrm{~h}$. Cells were released by washing three to five times with pre-warmed medium and plated into fresh DMEM, supplemented with $10 \%$ fetal bovine serum. Samples were taken at the indicated time points and processed for western blot analysis.

Immunoprecipitation and western blot assays. For immunoprecipitation assays, HEK 293T and HeLa cells were lysed in M-PER (Thermo Scientific) with protease inhibitors, including $1 \mathrm{mM} \mathrm{Na}_{3} \mathrm{VO}_{4}, 10 \mu \mathrm{g} \mathrm{ml}^{-1}$ aprotinin, $1 \mathrm{mM}$ pepstatin, $1 \mathrm{mM}$ leupeptin and $1 \mathrm{mM}$ phenylmethylsulphonyl fluoride (PMSF). Nocodazole $\left(10 \mu \mathrm{g} \mathrm{ml}^{-1}\right)$ was added to completely depolymerize microtubules. FLAG M2 beads (20 $\mu$; Sigma) were incubated with cell lysate for $1 \mathrm{~h}$ at $4{ }^{\circ} \mathrm{C}$ and washed three to five times in mammalian cell lysis buffer (50 mM HEPES, $100 \mathrm{mM} \mathrm{NaCl}, 1.5 \mathrm{mM}$ $\mathrm{MgCl}_{2}, 1 \mathrm{mM}$ EDTA, $1 \%$ Triton X-100 and 10\% glycerol). For endogenous immunoprecipitation assays, synchronized HeLa cells were shaken off and lysed. The cell lysate was incubated with $2 \mu \mathrm{g}$ rabbit IgG (Santa Cruz Biotechnology), NuSAP antibody 1:1,000 (ab93779, Abcam) or Kid antibody 1:1,000 (ab75783, Abcam) bound to protein A-Sepharose beads (Amersham Biosciences) for $1 \mathrm{~h}$ at $4{ }^{\circ} \mathrm{C}$. The immune complexes were washed three to five times in mammalian cell lysis buffer and applied to SDS-PAGE. Protein samples were separated on a $10 \%$ SDS gel and detected by the following primary antibodies: rabbit anti-FLAG 1:20,000 (F3165, Santa Cruz Biotechnology), rabbit anti-HA 1:2,000 (H6908, Santa Cruz Biotechnology), mouse anti-GAPDH 1:2,000 (sc32233, Santa Cruz Biotechnology), rabbit anti-cyclinB1 1:1,000 (H433, Santa Cruz Biotechnology), rabbit anti-Kid 1:1,000 (ab75783, Abcam), rabbit anti-NuSAP 1:1,000 (ab93779, Abcam) and mouse anti-NuSAP, which were detected by horseradish peroxidaseconjugated secondary antibodies (Santa Cruz Biotechnology).

Protein expression and GST pull-down. GST-Kid or His-NuSAP were expressed in Escherichia coli BL21 (DE3) pLysS at $20^{\circ} \mathrm{C}$ overnight after isopropyl- $\beta$-Dthiogalactoside (IPTG) induction. GST-Kid protein was purified with the use of glutathione-Sepharose 4B (Amersham Biosciences) and washed with wash buffer (50 mM Tris- $\mathrm{HCl}, 200 \mathrm{mM} \mathrm{NaCl}, 1 \mathrm{mM}$ EDTA and $1 \mathrm{mM}$ dithiothreitol (DTT)). His-NuSAP was resuspended in lysis buffer $\left(10 \mathrm{mM}\right.$ imidazole, $50 \mathrm{mM} \mathrm{NaH} \mathrm{NO}_{4}$, $300 \mathrm{mM} \mathrm{NaCl}, 1 \mathrm{mM}$ PMSF and protease inhibitor cocktail) and incubated with Ni-NTA agarose (Qiagen) at $4{ }^{\circ} \mathrm{C}$ for $1 \mathrm{~h}$. His-NuSAP protein was eluted with elution buffer $\left(250 \mathrm{mM}\right.$ imidazole, $50 \mathrm{mM} \mathrm{NaH} \mathrm{PO}_{4}$ and $\left.300 \mathrm{mM} \mathrm{NaCl}\right)$ and stored at $-80^{\circ} \mathrm{C}$. Kid-GFP-His and K560-Dendra2 were expressed in E. coli BL21 (DE3) pLysS at $16{ }^{\circ} \mathrm{C}$ overnight after IPTG induction. The bacterial cells were pelleted and resuspended in lysis buffer $(20 \mathrm{mM}$ imidazole, $100 \mathrm{mM} \mathrm{NaCl}$, $1 \mathrm{mM} \mathrm{MgCl} 2,0.1 \mathrm{mM}$ ATP, $1 \mathrm{mM}$ PMSF and protease inhibitor cocktail). After sonication, the lysates were cleared by centrifugation and the supernatants were incubated with Ni-NTA resin for $1 \mathrm{~h}$ at $4{ }^{\circ} \mathrm{C}$. Proteins were eluted with elution buffer containing $250 \mathrm{mM}$ imidazole and dialysed into storage buffer $(10 \mathrm{mM}$
PIPES, $100 \mathrm{mM} \mathrm{NaCl}, 1 \mathrm{mM} \mathrm{MgCl} 2,0.01 \mathrm{mM}$ ATP, $1 \mathrm{mM}$ EGTA and $1 \mathrm{mM}$ DTT). The protein was aliquoted and stored at $-80^{\circ} \mathrm{C}$. The in vitro GST pull-down assay using purified proteins was performed in $50 \mathrm{mM}$ Tris- $\mathrm{HCl}, \mathrm{pH} 7.0,150 \mathrm{mM} \mathrm{NaCl}$, $1 \mathrm{mM}$ EGTA and $0.5 \%$ Triton X-100, with $2 \mu \mathrm{g}$ GST fusion protein and $2 \mu \mathrm{g}$ target protein. After 3-h incubation at $4{ }^{\circ} \mathrm{C}$, the GST beads were washed three times, and protein samples were separated using SDS-PAGE and analysed with Coomassie blue staining.

Microtubule gliding assay. Motility assays were performed as previously described ${ }^{57}$. Rhodamine-labelled microtubules (Cytoskeleton) were polymerized in BRB80 buffer (80 mM PIPES, $1 \mathrm{mM} \mathrm{MgCl}_{2}$ and $1 \mathrm{mM}$ EGTA) containing $1 \mathrm{mM}$ GTP at $37^{\circ} \mathrm{C}$ for $10 \mathrm{~min}$. Flow chambers with a volume of $\sim 10 \mu \mathrm{l}$ were constructed with adhesive tapes on glass slides and incubated with $1 \mathrm{mg} \mathrm{ml}^{-1}$ casein (Sigma) and kinesin $(50 \mu \mathrm{M}$ for Kid and $20 \mu \mathrm{M}$ for K560). MTs were diluted in BRB80 buffer with $0.5 \mathrm{mg} \mathrm{ml}^{-1}$ casein, $22.5 \mathrm{mM}$ glucose, $0.22 \mathrm{mg} \mathrm{ml}^{-1}$ glucose oxidase, $0.036 \mathrm{mg} \mathrm{ml}^{-1}$ catalase, $3 \mathrm{mM}$ ATP and $10 \mu \mathrm{M}$ taxol (Sigma), and were introduced into the flow chamber. MTs were incubated in the presence or absence of $20 \mathrm{nM}$ NuSAP protein at room temperature for $5 \mathrm{~min}$ before flowing into the chamber. Unbound microtubules were washed off and images were collected near the middle of the flow cell using the Ultraview Vox Spinning disc confocal system (PerkinElmer).

Immunofluorescence. HeLa cells were cultured on ethanol-sterilized coverslips in 12-well plates and were synchronized or monastrol-treated before fixation with ice-cold methanol at $-20^{\circ} \mathrm{C}$ for $10 \mathrm{~min}$, followed by rehydration with $1 \times \mathrm{PBS}$ at room temperature for $10 \mathrm{~min}$. The fixed cells were permeabilized with $0.3 \%$ Triton X-100 in $1 \times$ PBS for 15 min and blocked with $2 \%$ bovine serum albumin for $45 \mathrm{~min}$ at room temperature. Mouse anti- $\alpha$-tubulin 1:2,000 (T9026, Sigma), rabbit anti- $\gamma$-tubulin 1:200 (T5192, Sigma), human anticentromere antibody 1:2,000 (HSM0101, ImmunoVision), rabbit anti-Kid 1:200 (ab75783, Abcam) and rabbit anti-NuSAP 1:200 (ab93779, Abcam) were utilized to stain the respective proteins in cells. Alexa Fluor dye-conjugated goat anti-mouse, goat anti-rabbit or goat anti-human IgG (Invitrogen) were used as the secondary antibodies. DNA was stained with Hoechst 33342 (Invitrogen). Cells were mounted in FluorSave reagent (Calbiochem). The images were processed using either the Volocity software (PerkinElmer) or Image J (National Institutes of Health, Bethesda, MD), as required.

Live-cell imaging. Stable H2B-mCherry HeLa cells were cultured on 35-mm glass-bottom Petri dishes (Greiner Bio-One) and synchronized or monastroltreated before imaging. Images were collected at $37^{\circ} \mathrm{C}$ using the Ultraview Vox Spinning disc confocal system (PerkinElmer), which includes a CSD-X1 spinning disk head (Yokogawa), a solid-state diode laser with both diode and diode pumped solid-state (DPPS) modules (25-50 mM) and an EMCCD camera C9100-50 (Hamamatsu). The images were taken with an Olympus Uplan SApo $100 \times 1.4$ oil lens controlled with the Volocity software (PerkinElmer).

Previous studies have indicated that the movement of centromeres at the periphery of the spindle is different from that of the KT in the middle $e^{7,58,59}$. The imaging conditions were based on the phototoxic effects on the cells and KT detection quality, in particular for the 3D live-cell imaging ${ }^{36,60,61}$; thus, only the centromeres in the middle region of the spindle were imaged and analysed. It has previously been suggested that to avoid aliasing in the analysis of motion characteristics, the motion should be sampled at least two times faster than its frequency ${ }^{62}$. The KT oscillation in HeLa cells is $\sim 60$ s per period, which would require a sampling interval less than $30 \mathrm{~s}$. To accommodate these requirements, $0.5 \mu \mathrm{m}$ of each $z$ section for $3 \mu \mathrm{m}$ within the centre of the spindle was acquired every $15 \mathrm{~s}$ for $10 \mathrm{~min}$. Only cells with spindles oriented parallel to the plane of focus were analysed and the focal plane at the centre of the spindle was chosen.

For FRAP experiments, stable mCherry- $\alpha$-tubulin HeLa cells were synchronized at the metaphase stage and imaged in a $37^{\circ} \mathrm{C}$ humid chamber supplied with $5 \% \mathrm{CO}_{2}$ with a UltraVIEW Vox spinning disc confocal system (PerkinElmer). A $1 \times 4 \mu \mathrm{m}$ photobleaching spot was placed near the middle of the spindle. Fluorescence intensities were photobleaching- and background-corrected using the Volocity 3D imaging software (PerkinElmer). Double exponential regression analysis in the Sigmaplot software (Jandel Scientific) was utilized to fit the data to the equation $F(t)=A_{1} e^{-k 1 t}+A_{2} e^{-k 2 t}$, where $F(t)$ stands for fluorescence intensity over time, and $k_{1}$ and $k_{2}$ represent the proportion of iMTs with a fast phase and kMTs with a slow phase, respectively. MT turnover half-life time was calculated by the equation $T_{1 / 2}=\operatorname{Ln} 2 / k$ (refs 43,63). For photoactivation, HeLa cells transfected with PAGFP- $\alpha$-tubulin were synchronized and a $405-\mathrm{nm}$ laser was focused on the selected $1 \times 4 \mu \mathrm{m}$ area for $1 \mathrm{~s}$. Fluorescence intensity of the activated region was photobleaching- and background-corrected using Volocity (PerkinElmer), and the half-lives of kMTs and iMTs were analysed by double exponential fitting as described ${ }^{42,43,64}$.

Quantitative analyses of 3D chromosome movements. The 3D images were first background-corrected with the background image in the Volocity software (PerkinElmer), and noise removal was achieved by convolving the raw 
images with the 3D Gaussian filter in Imaris (Bitplane). Chromosomes were tracked using the Imaris (Bitplane) 3D surface detection function, followed by manual corrections. The velocity and acceleration of chromosomes were analysed with Imaris using a centre projection function, and the images were processed with the Volocity software (PerkinElmer) and Image J (National Institutes of Health).

Quantitative analyses of 3D centromere oscillation. To correctly detect the centromeres, the images were first background-corrected with the background image in the Volocity software (PerkinElmer), and noise removal was achieved by convolving the raw images with the 3D Gaussian filter in Imaris (Bitplane). Centromeres in bipolar metaphase cells and monopolar cells were tracked using the Imaris (Bitplane) 3D spot detection function, followed by manual corrections. The centromere trajectories were constructed using the single-particle tracking algorithm within the centre of the spindle to avoid track interruptions resulting from temporary centromere disappearance by movement out of the imaging volume, or detection failure. Tracking was detected with a Brownian motion model because centromere oscillation is neither linear nor contains any merging and splitting events. On the basis of the approximate parameters of the centromere movement, we set the maximum gap-closing time window as 1 and the search radius upper limit as 1.5. Only the tracks with more than 20 time points detected were analysed. To correct cell movement during the process of imaging, KT positions $(x, y, z, t)$ were registered to the relative 3D position compared with spindle pole $\left(x_{\mathrm{o}}, y_{\mathrm{o}}, z_{\mathrm{o}}, t\right)$ over time as $\left(x-x_{\mathrm{o}}, y-y_{\mathrm{o}}, z-z_{\mathrm{o}}, t\right)$.

The 3D position information of each centromere track was extracted from Imaris, and the data were analysed with custom scripts in Perl (Perl Inc.) and run from the Perl command line. Sister pairing was based on the average distance and the distance variation using both $3 \mathrm{D}$ spatial and temporal information of the centromere tracks ${ }^{35}$. The average of the sister track pair should be closer and the distance variation within each track should be smaller, compared with other non-sisters. Thus, we calculated the average distance $d_{\mathrm{ij}}$ $\left(d_{\mathrm{ij}, \mathrm{t}}=\sqrt{\left(x_{\mathrm{i}, \mathrm{t}}-x_{\mathrm{j}, \mathrm{t}}\right)^{2}+\left(y_{\mathrm{i}, \mathrm{t}}-y_{\mathrm{j}, \mathrm{t}}\right)^{2}+\left(z_{\mathrm{i}, \mathrm{t}}-z_{\mathrm{j}, \mathrm{t}}\right)^{2}}, d_{\mathrm{ij}}=\frac{1}{n} \sum_{t=1}^{n} d_{\mathrm{ij}, \mathrm{t}}\right)$ and the variance of the distance $v_{\mathrm{ij}}\left(v_{\mathrm{ij}}=\frac{1}{n-1} \sum_{t=1}^{n}\left(d_{\mathrm{ij}, \mathrm{t}}-d_{\mathrm{ij}}\right)^{2}\right)$ between each pair $\mathrm{i}\left(x_{i}, y_{\mathrm{i}}\right.$, $\left.z_{\mathrm{i}}, t\right)$ and $\mathrm{j}\left(x_{\mathrm{j}}, y_{\mathrm{j}}, z_{\mathrm{j}}, t\right)$ tracks over the course of the imaging. The cost of the sister pairing considers both criteria together and was defined as $d_{\mathrm{ij}} \times v_{\mathrm{ij}}$. The acceptable bounds were set as 1.5 , and the combination of the trajectory pairings was taken with the minimum global cost as the assignment of the sister tracks. Only paired tracks with over 20 time points matched out of the total 41 time points were analysed. The ICD was calculated as the average distance between the sister pairs $\left(\operatorname{ICD}_{\mathrm{ij}, \mathrm{t}}=\sqrt{\left(x_{\mathrm{i}, \mathrm{t}}-x_{\mathrm{j}, \mathrm{t}}\right)^{2}+\left(y_{\mathrm{i}, \mathrm{t}}-y_{\mathrm{j}, \mathrm{t}}\right)^{2}+\left(z_{\mathrm{i}, \mathrm{t}}-z_{\mathrm{j}, \mathrm{t}}\right)^{2}}, \mathrm{ICD}=\overline{\operatorname{ICD}_{\mathrm{ij}, \mathrm{t}}}\right)$.

The direction of the movement was set based on the change of the distances between each centromere in the sister tracks to the spindle pole $\left(x_{\mathrm{o}}, y_{\mathrm{o}}, z_{\mathrm{o}}, t\right)$ during two time points $\left(\mathrm{dp}_{\mathrm{i}, \mathrm{t}}=\sqrt{\left(x_{\mathrm{i}, \mathrm{t}}-x_{\mathrm{o}, \mathrm{t}}\right)^{2}+\left(y_{\mathrm{i}, \mathrm{t}}-y_{\mathrm{o}, \mathrm{t}}\right)^{2}+\left(z_{\mathrm{i}, \mathrm{t}}-z_{\mathrm{o}, \mathrm{t}}\right)^{2}}\right)$. In bipolar cells, in a sister pair $\mathrm{i}\left(x_{\mathrm{i}}, y_{\mathrm{i}}, z_{\mathrm{i}}, t\right)$ and $\mathrm{j}\left(x_{\mathrm{j}}, y_{\mathrm{j}}, z_{\mathrm{j}}, t\right)$, for centromere $\mathrm{i}$, which is closer to the specified spindle pole $\left(\mathrm{dp}_{\mathrm{i}}<\mathrm{dp}_{\mathrm{j}}\right)$, the sign was set as positive $\left(\operatorname{Sign}_{\mathrm{i}, \mathrm{t}}=+1\right)$ for movement to the pole $\left(\mathrm{dp}_{\mathrm{i}, \mathrm{t}}>\mathrm{dp}_{\mathrm{i}, \mathrm{t}+1}\right)$ and negative $\left(\operatorname{Sign}_{\mathrm{i}, \mathrm{t}}=-1\right)$ for movement away from the pole $\left(\mathrm{dp}_{\mathrm{i}, \mathrm{t}}<\mathrm{dp}_{\mathrm{i}, \mathrm{t}+1}\right)$. For the other centromere, $\mathrm{j}$, which is further from the specified spindle pole, the sign was the opposite $\left(\operatorname{Sign}_{\mathrm{j}, \mathrm{t}}=+1\right)$ for movement away from the pole $\left(\mathrm{dp}_{\mathrm{j}, \mathrm{t}}<\mathrm{dp}_{\mathrm{j}, \mathrm{t}+1}\right)$ and negative $\left(\operatorname{Sign}_{\mathrm{j}, \mathrm{t}}=-1\right)$ for movement to the pole $\left(\mathrm{dp}_{\mathrm{j}, \mathrm{t}}>\mathrm{dp}_{\mathrm{j}, \mathrm{t}+1}\right)$. In monopolar cells, the sign was set as positive $\left(\operatorname{Sign}_{\mathrm{m}, \mathrm{t}}=+1\right)$ for movement to the pole $\left(\mathrm{dp}_{\mathrm{m}, \mathrm{t}}>\mathrm{dp} \mathrm{p}_{\mathrm{t}, \mathrm{t}}\right)$ and negative $\left(\operatorname{Sign}_{\mathrm{m}, \mathrm{t}}=-1\right)$ for movement away from the pole $\left(\mathrm{dp}_{\mathrm{m}, \mathrm{t}}<\mathrm{dp} \mathrm{m}, \mathrm{t}+1\right)$. The halfperiod was calculated as the time between two directional switching points based on the sign change (if $\operatorname{Sign}_{\text {switch }+1} \neq \operatorname{Sign}_{\text {switch }}, T_{\text {half }}=\frac{1}{n} \sum_{1}^{n}\left(t_{\text {switch }+1}-t_{\text {switch }}\right)$ ).

The mimic centre track of sister pair $\mathrm{i}\left(x_{\mathrm{i}}, y_{\mathrm{i}}, z_{\mathrm{i}}, t\right)$ and $\mathrm{j}\left(x_{\mathrm{j}}, y_{\mathrm{j}}, z_{\mathrm{j}}, t\right)$ was set as $\left(\left(x_{\mathrm{i}}+x_{\mathrm{j}}\right) / 2,\left(y_{\mathrm{i}}+y_{\mathrm{j}}\right) / 2,\left(z_{\mathrm{i}}+z_{\mathrm{j}}\right) / 2, t\right)$. The oscillation amplitude was set as the distance of the projected centre moved between directional switch points

$\left(\mathrm{Amp}=\frac{1}{n} \sqrt{\sum_{1}^{n}\left(x_{\text {switch }+1}-x_{\text {switch }}\right)^{2}+\left(y_{\text {switch }+1}-y_{\text {switch }}\right)^{2}+\left(z_{\text {switch }+1}-z_{\text {switch }}\right)^{2}}\right.$, Amp $\left.=\overline{\mathrm{Amp}_{t}}\right)$. The average $\left(V_{t}=\frac{1}{\Delta t} \sqrt{\left(x_{t+1}-x_{t}\right)^{2}+\left(y_{t+1}-y_{t}\right)^{2}+\left(z_{t+1}-z_{t}\right)^{2}}\right.$, $\left.V=\bar{V}_{t}\right)$ and the distribution of the centre velocity were calculated by the absolute value. The sign of the presented centre velocity over time shows the direction of the centre oscillation. The sign was set as positive $\left(\operatorname{Sign}_{\mathrm{c}, \mathrm{t}}=+1\right)$ for movement to the pole $\left(\mathrm{dp}_{\mathrm{c}, \mathrm{t}}>\mathrm{dp}_{\mathrm{c}, \mathrm{t}+1}\right)$ and negative $\left(\operatorname{Sign}_{\mathrm{c}, \mathrm{t}}=-1\right)$ for movement away from the pole $\left(\mathrm{dp}_{c, t}<d \mathrm{p}_{c, t+1}\right)$. The statistical analysis for calculation of the standard error of mean and confidence is described ${ }^{62}$ as $S^{2}=\frac{1}{n-1} \sum_{i=1}^{n}\left(\mathrm{X}_{\mathrm{i}}-\bar{X}\right)^{2} \quad$ s.e.m. $=\frac{S}{\sqrt{n}}$.

\section{References}

1. Lewis, W. H. Changes of viscosity and cell activity. Science 89, 400 (1939)

2. Skibbens, R. V., Skeen, V. P. \& Salmon, E. D. Directional instability of kinetochore motility during chromosome congression and segregation in mitotic newt lung cells: a push-pull mechanism. J. Cell Biol. 122, 859-875 (1993)

3. Hays, T. S. \& Salmon, E. D. Poleward force at the kinetochore in metaphase depends on the number of kinetochore microtubules. J. Cell Biol. 110, 391-404 (1990).
4. Rieder, C. L., Davison, E. A., Jensen, L. C., Cassimeris, L. \& Salmon, E. D. Oscillatory movements of monooriented chromosomes and their position relative to the spindle pole result from the ejection properties of the aster and half-spindle. J. Cell Biol. 103, 581-591 (1986).

5. Levesque, A. A. \& Compton, D. A. The chromokinesin Kid is necessary for chromosome arm orientation and oscillation, but not congression, on mitotic spindles. J. Cell Biol. 154, 1135-1146 (2001).

6. Stumpff, J., von Dassow, G., Wagenbach, M., Asbury, C. \& Wordeman, L. The kinesin-8 motor Kif18A suppresses kinetochore movements to control mitotic chromosome alignment. Dev. cell 14, 252-262 (2008).

7. Stumpff, J., Wagenbach, M., Franck, A., Asbury, C. L. \& Wordeman, L. Kif18A and chromokinesins confine centromere movements via microtubule growth suppression and spatial control of kinetochore tension. Dev. cell 22, 1017-1029 (2012).

8. Wandke, C. et al. Human chromokinesins promote chromosome congression and spindle microtubule dynamics during mitosis. J. Cell Biol. 198, 847-863 (2012).

9. Joglekar, A. P. \& Hunt, A. J. A simple, mechanistic model for directional instability during mitotic chromosome movements. Biophys. J. 83, 42-58 (2002).

10. Liu, J., Desai, A., Onuchic, J. N. \& Hwa, T. A mechanobiochemical mechanism for monooriented chromosome oscillation in mitosis. Proc. Natl Acad. Sci. USA 104, 16104-16109 (2007).

11. Vladimirou, E., Harry, E., Burroughs, N. \& McAinsh, A. D. Springs, clutches and motors: driving forward kinetochore mechanism by modelling. Chromosome Res. 19, 409-421 (2011).

12. Sutradhar, S. \& Paul, R. Tug-of-war between opposing molecular motors explains chromosomal oscillation during mitosis. J. Theor. Biol. 344, 56-69 (2014).

13. Maiato, H., Sampaio, P. \& Sunkel, C. E. Microtubule-associated proteins and their essential roles during mitosis. Int. Rev. Cytol. 241, 53-153 (2004).

14. Raemaekers, T. et al. NuSAP, a novel microtubule-associated protein involved in mitotic spindle organization. The Journal of cell biology 162, 1017-1029 (2003).

15. Ribbeck, K. et al. NuSAP, a mitotic RanGTP target that stabilizes and crosslinks microtubules. Mol. Biol. Cell 17, 2646-2660 (2006).

16. Ribbeck, K., Raemaekers, T., Carmeliet, G. \& Mattaj, I. W. A role for NuSAP in linking microtubules to mitotic chromosomes. Curr. Biol. 17, 230-236 (2007).

17. Hussain, S. et al. The nucleolar RNA methyltransferase Misu (NSun2) is required for mitotic spindle stability. J. Cell Biol. 186, 27-40 (2009).

18. Li, L. et al. NuSAP is degraded by APC/C-Cdh1 and its overexpression results in mitotic arrest dependent of its microtubules' affinity. Cell. Signal. 19, 2046-2055 (2007).

19. Song, L. \& Rape, M. Regulated degradation of spindle assembly factors by the anaphase-promoting complex. Molecular cell 38, 369-382 (2010).

20. Fujiwara, T. et al. Expression analyses and transcriptional regulation of mouse nucleolar spindle-associated protein gene in erythroid cells: essential role of NF-Y. British journal of haematology 135, 583-590 (2006).

21. Gulzar, Z. G., McKenney, J. K. \& Brooks, J. D. Increased expression of NuSAP in recurrent prostate cancer is mediated by E2F1. Oncogene 32, 70-77 (2013).

22. Iyer, J., Moghe, S., Furukawa, M. \& Tsai, M. Y. What's Nu(SAP) in mitosis and cancer? Cell. Signal. 23, 991-998 (2011).

23. Kokkinakis, D. M., Liu, X. \& Neuner, R. D. Modulation of cell cycle and gene expression in pancreatic tumor cell lines by methionine deprivation (methionine stress): implications to the therapy of pancreatic adenocarcinoma. Mol. Cancer Ther. 4, 1338-1348 (2005).

24. Wadia, P. P. et al. Antibodies specifically target AML antigen NuSAP1 after allogeneic bone marrow transplantation. Blood 115, 2077-2087 (2010).

25. Xie, P. et al. ATM-mediated NuSAP phosphorylation induces mitotic arrest. Biochem. Biophys. Res. Commun. 404, 413-418 (2011).

26. Heald, R. Motor function in the mitotic spindle. Cell 102, 399-402 (2000).

27. Mazumdar, M. \& Misteli, T. Chromokinesins: multitalented players in mitosis Trends Cell Biol. 15, 349-355 (2005).

28. Vanneste, D., Ferreira, V. \& Vernos, I. Chromokinesins: localization-dependent functions and regulation during cell division. Biochem. Soc. Trans. 39, 1154-1160 (2011).

29. Tokai, N. et al. Kid, a novel kinesin-like DNA binding protein, is localized to chromosomes and the mitotic spindle. EMBO J. 15, 457-467 (1996).

30. Yajima, J. et al. The human chromokinesin Kid is a plus end-directed microtubule-based motor. EMBO J. 22, 1067-1074 (2003).

31. Trieselmann, N., Armstrong, S., Rauw, J. \& Wilde, A. Ran modulates spindle assembly by regulating a subset of TPX2 and Kid activities including Aurora A activation. J. Cell Sci. 116, 4791-4798 (2003).

32. Levesque, A. A., Howard, L., Gordon, M. B. \& Compton, D. A. A functional relationship between NuMA and kid is involved in both spindle organization and chromosome alignment in vertebrate cells. Mol. Biol. Cell 14, 3541-3552 (2003). 
33. Santamaria, A., Nagel, S., Sillje, H. H. \& Nigg, E. A. The spindle protein CHICA mediates localization of the chromokinesin Kid to the mitotic spindle. Curr. Biol. 18, 723-729 (2008).

34. Cane, S., Ye, A. A., Luks-Morgan, S. J. \& Maresca, T. J. Elevated polar ejection forces stabilize kinetochore-microtubule attachments. J. Cell Biol. 200, 203-218 (2013).

35. Shiroguchi, K., Ohsugi, M., Edamatsu, M., Yamamoto, T. \& Toyoshima, Y. Y. The second microtubule-binding site of monomeric kid enhances the microtubule affinity. J. Biol. Chem. 278, 22460-22465 (2003).

36. Jaqaman, K. et al. Kinetochore alignment within the metaphase plate is regulated by centromere stiffness and microtubule depolymerases. J. Cell Biol. 188, 665-679 (2010).

37. Ke, K., Cheng, J. \& Hunt, A. J. The distribution of polar ejection forces determines the amplitude of chromosome directional instability. Curr. Biol. 19, 807-815 (2009).

38. Wan, X., Cimini, D., Cameron, L. A. \& Salmon, E. D. The coupling between sister kinetochore directional instability and oscillations in centromere stretch in metaphase PtK1 cells. Mol. Biol. Cell 23, 1035-1046 (2012).

39. Cai, S., O'Connell, C. B., Khodjakov, A. \& Walczak, C. E. Chromosome congression in the absence of kinetochore fibres. Nat. Cell Biol. 11, 832-838 (2009).

40. DeLuca, J. G. et al. Nuf2 and Hecl are required for retention of the checkpoint proteins Mad1 and Mad2 to kinetochores. Curr. Biol. 13, 2103-2109 (2003).

41. DeLuca, J. G. et al. Hec1 and nuf2 are core components of the kinetochore outer plate essential for organizing microtubule attachment sites. Mol. Biol. Cell 16, 519-531 (2005).

42. Kabeche, L. \& Compton, D. A. Cyclin A regulates kinetochore microtubules to promote faithful chromosome segregation. Nature 502, 110-113 (2013).

43. Zhai, Y., Kronebusch, P. J. \& Borisy, G. G. Kinetochore microtubule dynamics and the metaphase-anaphase transition. J. Cell Biol. 131, 721-734 (1995).

44. Feine, O., Zur, A., Mahbubani, H. \& Brandeis, M. Human Kid is degraded by the APC/C(Cdh1) but not by the APC/C(Cdc20). Cell Cycle 6, 2516-2523 (2007).

45. Tahara, K. et al. Importin-beta and the small guanosine triphosphatase Ran mediate chromosome loading of the human chromokinesin Kid. J. Cell Biol. 180, 493-506 (2008).

46. Brouhard, G. J. \& Hunt, A. J. Microtubule movements on the arms of mitotic chromosomes: polar ejection forces quantified in vitro. Proc. Natl Acad. Sci. USA 102, 13903-13908 (2005).

47. King, J. M., Hays, T. S. \& Nicklas, R. B. Dynein is a transient kinetochore component whose binding is regulated by microtubule attachment, not tension. J. Cell Biol. 151, 739-748 (2000).

48. Wordeman, L., Wagenbach, M. \& von Dassow, G. MCAK facilitates chromosome movement by promoting kinetochore microtubule turnover. J. Cell Biol. 179, 869-879 (2007).

49. Kline-Smith, S. L., Khodjakov, A., Hergert, P. \& Walczak, C. E. Depletion of centromeric MCAK leads to chromosome congression and segregation defects due to improper kinetochore attachments. Mol. Biol. Cell 15, 1146-1159 (2004).

50. Hunter, A. W. et al. The kinesin-related protein MCAK is a microtubule depolymerase that forms an ATP-hydrolyzing complex at microtubule ends. Mol. Cell 11, 445-457 (2003).

51. Wordeman, L. \& Mitchison, T. J. Identification and partial characterization of mitotic centromere-associated kinesin, a kinesin-related protein that associates with centromeres during mitosis. J. Cell Biol. 128, 95-104 (1995).

52. Mayr, M. I. et al. The human kinesin Kif18A is a motile microtubule depolymerase essential for chromosome congression. Curr. Biol. 17, 488-498 (2007).

53. McClelland, S. E. et al. The CENP-A NAC/CAD kinetochore complex controls chromosome congression and spindle bipolarity. EMBO J. 26, 5033-5047 (2007).

54. Friedman, J. R., Webster, B. M., Mastronarde, D. N., Verhey, K. J. \& Voeltz, G. K. ER sliding dynamics and ER-mitochondrial contacts occur on acetylated microtubules. J. Cell Biol. 190, 363-375 (2010).

55. DeLuca, J. G., Moree, B., Hickey, J. M., Kilmartin, J. V. \& Salmon, E. D. hNuf2 inhibition blocks stable kinetochore-microtubule attachment and induces mitotic cell death in HeLa cells. J. Cell Biol. 159, 549-555 (2002).

56. Nam, H. S. \& Benezra, R. High levels of Id1 expression define B1 type adult neural stem cells. Cell Stem Cell 5, 515-526 (2009).

57. Woehlke, G. et al. Microtubule interaction site of the kinesin motor. Cell 90, 207-216 (1997).

58. Cimini, D., Cameron, L. A. \& Salmon, E. D. Anaphase spindle mechanics prevent mis-segregation of merotelically oriented chromosomes. Curr. Biol. 14, 2149-2155 (2004).

59. Civelekoglu-Scholey, G. et al. Dynamic bonds and polar ejection force distribution explain kinetochore oscillations in PtK1 cells. J. Cell Biol. 201, 577-593 (2013).

60. Kitajima, T. S., Ohsugi, M. \& Ellenberg, J. Complete kinetochore tracking reveals error-prone homologous chromosome biorientation in mammalian oocytes. Cell 146, 568-581 (2011).

61. Magidson, V. et al. The spatial arrangement of chromosomes during prometaphase facilitates spindle assembly. Cell 146, 555-567 (2011).

62. Dorn, J. F., Danuser, G. \& Yang, G. Computational processing and analysis of dynamic fluorescence image data. Methods Cell Biol. 85, 497-538 (2008).

63. DeLuca, J. G. Kinetochore-microtubule dynamics and attachment stability. Methods Cell Biol. 97, 53-79 (2010).

64. Ye, F. et al. HURP regulates chromosome congression by modulating kinesin Kif18A function. Curr. Biol. 21, 1584-1591 (2011).

\section{Acknowledgements}

We thank the members of Y.-C.L laboratory for valuable discussion. We are also gratefu to Benjamin Walker, Cynthia Y.C. He, Cheng-Han Yu, Artem Yefremov, Chen Shi and Yusuke Toyama for helpful discussion and suggestions. We thank the Center of BioImaging Sciences (CBIS) at the National University of Singapore, especially to Ms Yan Tong for the technical support. In addition, we particularly thank Jared C. Cochran for kindly providing the K560 plasmids. We thank Lisa Cameron from Confocal and Light Microscope Core at the Dana Faber Cancer Institute for technical support. We also thank Xiaobin Mo and Shengping Xiao for helping protein expression. In addition, we thank Kerry McLaughlin of Insight Editing London for critical review. This work is financially supported by grants the Ministry of Education, Tier2 (MOE-T2-1-153; R-154-000-598-112) and Tier1 (R-154-000-571-112), as well as supported in part by NMRC-CBGR (R-154-000-637-511), Singapore to Y.-C.L.

\section{Author contributions}

C.L. and Y.-C.L. conceived and designed the research. C.L., C.X. and Q.Y. performed the experiments. C.L., B.C.L. and Y.-C.L. analysed data and wrote the manuscript.

\section{Additional information}

Supplementary Information accompanies this paper at http://www.nature.com/ naturecommunications

Competing financial interests: The authors declare no competing financial interests.

Reprints and permission information is available online at http://npg.nature.com/ reprintsandpermissions/

How to cite this article: Li, C. et al. NuSAP governs chromosome oscillation by facilitating the Kid-generated polar ejection force. Nat. Commun. 7:10597 doi: 10.1038/ncomms10597 (2016).

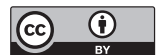

This work is licensed under a Creative Commons Attribution 4.0 International License. The images or other third party material in this article are included in the article's Creative Commons license, unless indicated otherwise in the credit line; if the material is not included under the Creative Commons license, users will need to obtain permission from the license holder to reproduce the material. To view a copy of this license, visit http://creativecommons.org/licenses/by/4.0/ 